\title{
Effects of frontal and plan solidities on aerodynamic parameters and the roughness sublayer in turbulent boundary layers
}

\author{
M. Placidi and B. Ganapathisubramani $\dagger$ \\ Aerodynamics and Flight Mechanics Research Group \\ Faculty of Engineering and the Environment \\ University of Southampton, SO17 1BJ, UK
}

(Received ?; revised ?; accepted ?. - To be entered by editorial office)

Experiments were conducted in the fully-rough regime on surfaces with large relative roughness height $(h / \delta \approx 0.1$, where $h$ is the roughness height and $\delta$ is the boundary layer thickness). The surfaces were generated by distributed LEGO ${ }^{\mathrm{TM}}$ bricks of uniform height, arranged in different configurations. Measurements were made with both floating-element drag-balance and high-resolution particle image velocimetry on six configurations with different frontal solidity, $\lambda_{F}$, at fixed plan solidity, $\lambda_{P}$, and vice versa, for a total of twelve rough-wall cases. Results indicate that the drag reaches a peak value $\lambda_{F} \approx 0.21$ or a constant $\lambda_{P}=0.27$, whilst it monotonically decreases for increasing values of $\lambda_{P}$ for a fixed $\lambda_{F}=0.15$. This is in contrast with previous studies in the literature based on cube roughness that show a peak in drag for both $\lambda_{F}$ and $\lambda_{P}$ variations. The influence of surface morphology on the depth of the roughness sublayer (RSL) is also investigated. Its depth is found to be inversely proportional to the roughness length, $y_{0}$. A decrease in $y_{0}$ is usually accompanied by a thickening of the the RSL and vice-versa. Proper orthogonal decomposition analysis was also employed. The shapes of the most energetic modes calculated using the data across the entire boundary layer are found to be selfsimilar across the twelve rough-walls cases, however, when the analysis is restricted to the roughness sublayer, differences that depend on the wall morphology are apparent. Moreover, the energy content of the POD modes within the RSL suggest that the effect of increased frontal solidity is to redistribute the energy towards the larger-scales (i.e. larger portion of energy is within the first few modes) whilst the opposite is found for variation of plan solidity.

Key words:

\section{Introduction and background}

Surface roughness is found in abundance in natural environments and plays an important role in a variety of practical and engineering applications. Nevertheless, while rough-walls are of great importance, they are much less understood than their smoothwall counterpart (Jimenez 2004). It is well established that, compared to the law of the wall for smooth-walls, any rough surface morphology results in a downward shift in the logarithmic portion of the velocity profile. For a rough-wall boundary layer, the velocity

$\dagger$ Email address for correspondence: g.bharath@soton.ac.uk 


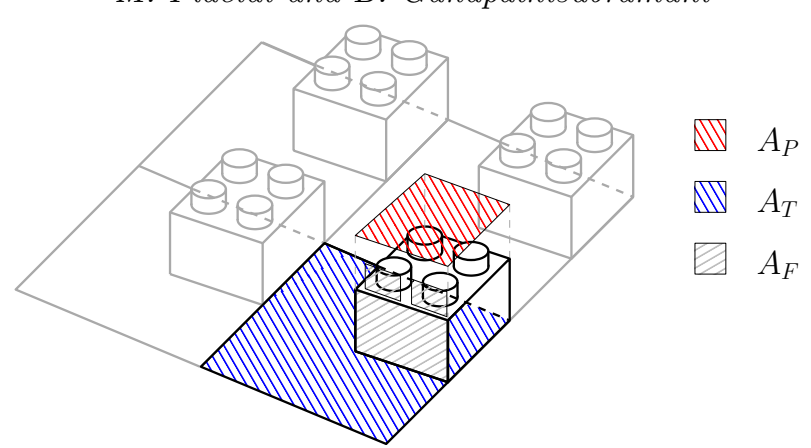

Figure 1. Definition of frontal and plan solidities. Black lines identify a single repeated unit, grey lines show how adjacent units are linked together to build the wall morphology. Inspired by Grimmond \& Oke (1999).

profile in the log-region can be expressed as:

$$
U^{+}=\frac{1}{\kappa} \ln \left(\frac{y-d}{y_{0}}\right) \equiv \frac{1}{\kappa} \ln (y-d)^{+}+B-\Delta U^{+},
$$

where $\kappa$ is the von Kármán constant and $B$ is the smooth-wall intercept. It is important to point out that the left-hand-side expression is only valid in fully-rough conditions (i.e. $y_{0}^{+}>2$ as from Castro 2007) where viscous effects at the surface are negligible, as in the current study. The downward shift of the log-region is represented by the roughness length $y_{0}$ in meteorology, or equivalently by $\Delta U^{+}$in the engineering community. $d$ is referred to as the zero-plane displacement, which Jackson (1981) proposed to be interpreted as the height at which the mean surface drag appears to act.

Early experimental studies of rough surfaces were focused on high-density rough-walls. For these wall morphologies, a good defining parameter was found to be the "equivalent sand roughness", $h_{s}$ (Nikuradse 1933). However, since Schlichting (1937), the tendency has been to characterise the effect of regularly distributed roughness using two density parameters: frontal and plan solidities. The frontal solidity, $\lambda_{F}$, is defined as the total projected frontal area of the roughness elements per unit wall-parallel area, while the plan solidity, $\lambda_{P}$, is the ratio between the plan area and the unit wall-parallel area, as highlighted in figure 1. It is important to highlight that this roughness characterisation is far from universal (i.e. valid for any rough-wall). As it is not in the intension of the authors to investigate the latter, previous studies are followed to facilitate comparisons and highlight some of the limitations of this formulation. In the current study, the roughness is classified via three parameters: $\lambda_{F}, \lambda_{P}, h / \delta$.

Various studies have examined the effect of surface morphology on drag, and attempted to find correlations for $y_{0}=f\left(\lambda_{F}, \lambda_{P}\right)$. These studies mainly classified the flow into two regimes: sparse $\left(\lambda_{F}<0.15\right)$, in which $y_{0}$ increases with solidity, and dense $\left(\lambda_{F} \geqslant 0.15\right)$, for which $y_{0}$ decreases. This is because, as the density increases so does the roughness of the system, but a point comes where adding new elements merely serves to reduce the effective drag of those already present due to mutual sheltering; that is, they start to smoothen the roughness of the system (Grimmond \& Oke 1999). Comprehensive reviews of different morphometric drag-prediction algorithms and an analysis of their accuracy can be found in Grimmond \& Oke (1999), Macdonald (1998) and more recently 
(a)

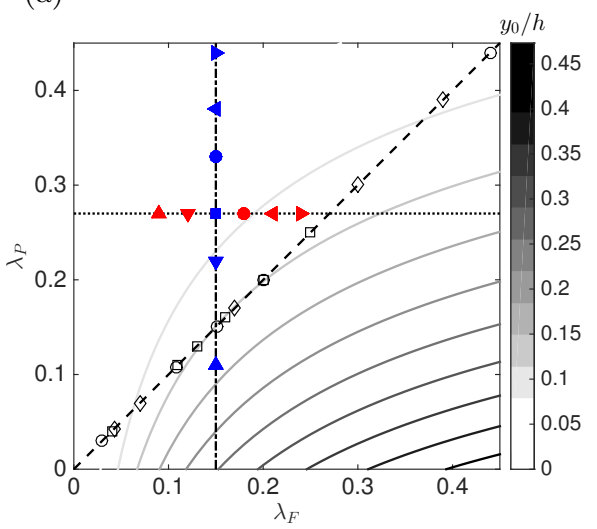

(b)

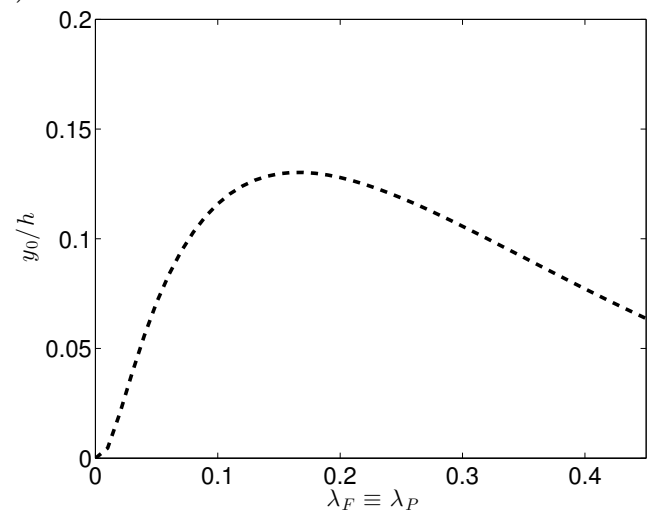

Figure 2. (a) Contour plot of the Macdonald's prediction of the roughness length behaviour as a function of $\lambda_{F}$ and $\lambda_{P}$ calculated using the expressions presented in Macdonald (1998) with $C d=1.2, k=0.41, \beta=1$ and $A=4.43$. Colorbar shows $y_{0} / h$. Dashed line represents $\lambda_{F} \equiv \lambda_{P}$ (cubes), dot-dashed line represents variation of $\lambda_{P}$ at fixed $\lambda_{F}$ and dotted line stands for variation of $\lambda_{F}$ at fixed $\lambda_{P}$. Filled symbols indicate the current experiment while empty symbols indicate respectively $\circ$ Kanda et al. (2004), $\diamond$ Hagishima et al. (2009) and $\square$ Leonardi \& Castro (2010). A cut along the dashed in figure $(a)$ is presented in $(b)$.

in Millward-Hopkins et al. (2011) and Kanda et al. (2013). The main limitation of these above-mentioned studies is the fact that the suggested correlations $y_{0}=y_{0}\left(\lambda_{F}, \lambda_{P}\right)$ are calculated over a collection of data sets from disparate sources with significantly different boundary conditions and characterised by geometrically different roughness elements. Regardless, amongst the morphometric bulk drag prediction methods, Macdonald's (1998) appears to be widely used as it incorporates a broader range of scenarios when compared to other methods (Grimmond \& Oke 1999 and Millward-Hopkins et al. 2011). Figure 2(a) shows its prediction for $y_{0}$ as a function of $\lambda_{F}$ and $\lambda_{P}$. Figure 2(b) shows the well-established empirical relationship between drag and $\lambda_{F}$. This has been observed in previous studies on rough-wall boundary layers as reported by Grimmond \& Oke (1999) and Jimenez (2004). However, the effect of plan solidity on bulk quantities is not clear. Additionally, effects of large roughness elements on these correlations remain unresolved. Castro (2007) pointed out that, although there is a substantial literature on rough-wall flows and there have been a number of attempts to develop useful correlations relating the surface friction to boundary-layer parameters over a wide range of surface roughness, such correlations have mainly considered only small $h / \delta$ and it is not known how adequate they are at larger values of $h / \delta$ or what the critical value $h / \delta$ should be.

Most previous studies that systematically explored the effect of surface morphology on drag were carried out with cubical roughness elements. These studies include both numerical and physical experiments (Cheng \& Castro 2002b; Coceal \& Belcher 2004; Kanda et al. 2004; Cheng et al. 2007; Hagishima et al. 2009; Santiago et al. 2008; Leonardi \& Castro 2010 among various others). Open symbols in figure 2(a) show the cases examined in these studies. Any conclusion regarding the relationship between plan solidity and drag deduced from these studies is limited, since for cube roughness, $\lambda_{F}$ is equal to $\lambda_{P}$. Similarly, any study on regular geometric staggered arrays of roughness for which the frontal and plan solidities are related by a mathematical relation (provided that there is no mutual sheltering between the elements) will lie on a similar curve to the dashed line in figure 2(a), although with a different slope. This means that the isolated effect of one of these two parameters will remain undetected. Isolating the effect of the two solidities 
is only possible by following the dotted line and the dot-dashed line (or any alternative lines parallel to those), i.e., varying $\lambda_{F}$ at a fixed $\lambda_{P}$ and vice-versa. These branches have not been explored before. In this study, we explore the behaviour of drag along those dotted and dot-dashed lines in figure $2(a)$ in turbulent boundary layers with relatively large roughness elements $(h / \delta \approx 0.1$, where $h$ is the height of the roughness element and $\delta$ is the local boundary layer thickness).

The aim of this study is to examine the individual effects of frontal and plan solidities at a constant $h / \delta \approx 0.1$ on drag and roughness sublayer. In order to do so, a systematic series of experiments were carried out by varying $\lambda_{F}$ at a fixed $\lambda_{P}$ and vice-versa. A total of twelve different configurations were tested and the resulting data, that covers the entire regime of solidities (sparse to dense), will be used to examine the drag generated by the different surfaces as well as their effect on the roughness sublayer. This will enable us to not only isolate the effects of $\lambda_{P}$ and $\lambda_{F}$ on the drag but also to examine the spatial similarity of the flow in case of strong roughness $(h / \delta \approx 0.1)$.

\section{Experimental Facility and Details}

\subsection{Experimental Facility}

The experiments were carried out in a suction wind tunnel at the University of Southampton. The same facility has been used for previous rough-wall studies such as Reynolds \& Castro (2008) and Amir \& Castro (2011). The tunnel has a working section of $4.5 \mathrm{~m}$ in length, with a $0.9 \mathrm{~m} \times 0.6 \mathrm{~m}$ cross-section. The free-stream turbulence intensity in the tunnel has been verified through hot wire anemometry measurements, to be homogenous across the cross-section and less than $0.3 \%$. In this study, $(x, y, z)$ are the streamwise, wall-normal and spanwise directions, respectively, with the plane $y=0$ being the bottom surface of the baseboard onto which the roughness elements were located, as shown in figure $3(a)$. The mean and fluctuating velocities along these three directions are denoted as $(U, V, W)$ and $(u, v, w)$, respectively. The point statistics presented in this paper are obtained by ensemble averaging over a number of PIV realisations followed by spatial averaging across the PIV field-of-view (FOV). Experiments were conducted in nominally zero-pressure-gradient $(\mathrm{ZPG})$ as the acceleration parameter $\left(K=\left(\nu / U_{e}^{2}\right)\left[d U_{e} / d x\right]\right)$ was less than $5 \times 10^{-8}$.

\subsection{Roughness morphology}

For rough surfaces, this study used LEGO ${ }^{\mathrm{TM}}$ baseboards onto which LEGO ${ }^{\mathrm{TM}}$ bricks (or blocks) were distributed in different patterns. Twelve different patterns were adopted in order to systematically examine the individual effects of frontal and plan solidities on the structure of the turbulence. Although not shown here for brevity, the effect of the incoming boundary layer (due to the baseboard) has been quantified. This was found to be negligible as the flow is in equilibrium and fully-developed over the bricks at the measurement location. Figure 3 shows the geometry of a LEGO ${ }^{\mathrm{TM}}$ element and the basic repetitive units adopted to generate the different patterns in analysis (frontal and plan solidities variations in figure $3(b)$ left and right respectively). A single dark square in the top view is a single LEGO $^{\text {TM }}$ brick. This brick has a streamwise $\times$ spanwise dimension of $7.8 \mathrm{~mm} \times 7.8 \mathrm{~mm}$ and its height is $h=11.4 \mathrm{~mm}$ (which includes the pin at the top) as shown in figure $3(a)$. Patterns LF1 to LF6 represent cases for which the frontal solidity is varied at a fixed plan solidity whilst, in cases LP1 to LP6 the plan solidity is varied at fixed frontal solidity. These variations follow the dotted and dot-dashed lines in figure $2(a)$. The different cases were designed on the basis of previous studies that show the location of the peaks in $y_{0}$ at $\lambda_{P}=0.27$ and $\lambda_{F}=0.15$ (Grimmond \& Oke 1999 and 
Jimenez 2004). Progressive repositioning of the downstream roughness elements in the sheltered regions of the upstream obstacles, has allowed us to achieve variations in plan solidity at fixed frontal solidity. The same sheltering principle is applied to obtain the cases with varying $\lambda_{F}$ at fixed $\lambda_{P}$. This has enabled the examination of the behaviour of the bulk quantities along the dotted and dot-dashed lines in figure 2(a). The filled symbols in 2(a) indicate the cases studied in this paper. The unit wall-parallel area of each repetitive units was also kept fixed at $70.2 \mathrm{~mm} \times 39 \mathrm{~mm}$ and $46.8 \mathrm{~mm} \times 46.8 \mathrm{~mm}$ for the frontal and plan variation cases respectively. In evaluating $\lambda_{F}$ and $\lambda_{P}$, the complete LEGO $^{\mathrm{TM}}$ bricks have been considered (including the pins on top of the blocks, as shown in figure 1).

\subsection{Floating-element drag-balance}

The drag generated by the different wall morphologies was directly measured via a floating element balance (which was based on the design of Krogstad \& Efros 2010). This balance was placed approximately $4 m$ downstream along the test section (the flow developed initially over the baseboard for $1.7 \mathrm{~m}$ and a further $2.3 \mathrm{~m}$ over the bricks). Figure 4 shows a schematic of the balance. A measurement patch (replica of the testing surface) is positioned in the tunnel floor through a cut hole; this element is mechanically connected to a two-arm lever system which converts the horizontal stress acting at the wall into a wall-normal load. A $370 \mathrm{~mm} \times 370 \mathrm{~mm}$ patch of the roughness was mounted on the top of the vertical arm of the balance to act as sensing element. The measuring element was centred in a hole cut at the bottom of the wind tunnel floor surrounded by approximately $1.5 \mathrm{~mm}$ gap to allow movement (i.e. readings onto the scale). It was important to prevent any possible airflow through this gap around the tile, as this would affect the measurements. As the present work is carried out in a suction wind tunnel, the static pressure inside the tunnel was always significantly lower than the atmospheric pressure. This necessitated mounting the entire balance in a sealed box, so that the measured force was not influenced. The sealed box was made out of soft plastic sheet. The static pressure inside both the tunnel and the box were monitored to prevent pressure difference effect. This was found to be negligible within the measurement uncertainty. The relatively big sensing element's size, compared to the original version in Krogstad \& Efros (2010), allows a number of repeated units to be included in the measurements patch. The lever system, which rests on a knife edge, allows mechanical amplification of the forces based on the mutual length of the arms. The normal load is then measured by a high-sensitivity off-the-shelf precision Ohaus scale with a sensitivity of $0.01 \mathrm{~g}$ (Gold Series no. TAJ602/A). The measurement resolution, in the worst case, was typically $2.5 \times 10^{-4}$ the applied load. For additional details on the balance, the reader is referred to the original design in Krogstad \& Efros (2010) and Efros (2011).

\subsection{Particle image velocimetry}

\subsubsection{Planar PIV}

Particle Image Velocimetry (PIV) measurements were taken for all patterns at a freestream velocity, $U_{\infty}$, of $11.5 \mathrm{~m} / \mathrm{s}$. The test location was at the same streamwise location as the skin-friction measurements. As will be shown later, at this freestream velocity the flow over all surfaces is considered to meet fully-rough conditions (or very close to it) to within the uncertainty of the skin-friction measurements. The flow was seeded with vaporised glycol-water solution particles (1 $\mu \mathrm{m}$ in diameter) illuminated with a $0.7 \mathrm{~mm}$ thick laser sheet produced by a pulsed New Wave Nd:YAG laser system operating at $200 \mathrm{~mJ}$. The position of the laser sheet with respect to the roughness configuration is indicated by dashed lines in figure $3(b)$. Streamwise wall-normal $(x, y)$ 
(a) Side view

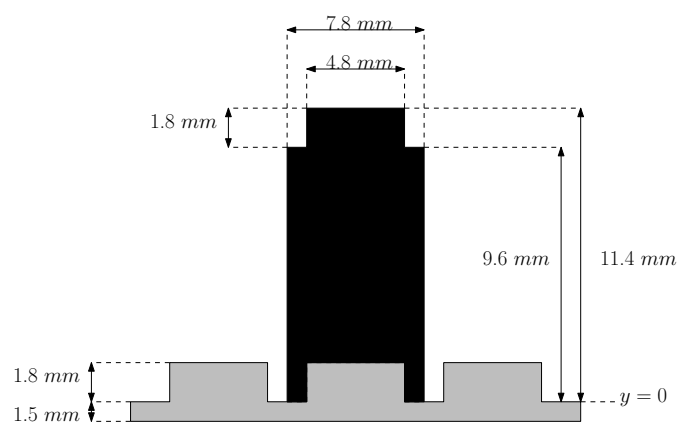

(b) Top View (basic square unit: $7.8 \times 7.8 \mathrm{~mm}^{2}$ )

\begin{tabular}{|c|c|}
\hline $\begin{array}{l}\text { Pattern: LF1 } \\
\lambda_{F}=0.09 \\
\lambda_{P}=0.27\end{array}$ & $\begin{array}{l}\text { Pattern: LP1 } \\
\lambda_{P}=0.11 \\
\lambda_{F}=0.15\end{array}$ \\
\hline $\begin{array}{l}\text { Pattern: LF2 } \\
\lambda_{F}=0.12 \\
\lambda_{P}=0.27\end{array}$ & $\begin{array}{l}\text { Pattern: LP2 } \\
\lambda_{P}=0.22 \\
\lambda_{F}=0.15\end{array}$ \\
\hline $\begin{array}{l}\text { Pattern: LF3 } \\
\lambda_{F}=0.15 \\
\lambda_{P}=0.27\end{array}$ & $\begin{array}{l}\text { Pattern: LP3 } \\
\lambda_{P}=0.27 \\
\lambda_{F}=0.15\end{array}$ \\
\hline $\begin{array}{l}\text { Pattern: LF4 } \\
\lambda_{F}=0.18 \\
\lambda_{P}=0.27\end{array}$ & $\begin{array}{l}\text { Pattern: LP4 } \\
\lambda_{P}=0.33 \\
\lambda_{F}=0.15\end{array}$ \\
\hline $\begin{array}{l}\text { Pattern: LF5 } \\
\lambda_{F}=0.21 \\
\lambda_{P}=0.27\end{array}$ & $\begin{array}{l}\text { Pattern: LP5 } \\
\lambda_{P}=0.39 \\
\lambda_{F}=0.15\end{array}$ \\
\hline $\begin{array}{l}\text { Pattern: LF6 } \\
\lambda_{F}=0.24 \\
\lambda_{P}=0.27\end{array}$ & $\begin{array}{l}\text { Pattern: LP6 } \\
\lambda_{P}=0.44 \\
\lambda_{F}=0.15\end{array}$ \\
\hline
\end{tabular}

Figure 3. (a) LEGO ${ }^{\mathrm{TM}}$ brick geometry, (b) roughness elements' patterns with varying $\lambda_{F}$ at $\lambda_{P}=$ const $=0.27$ (left) and roughness elements' patterns with varying $\lambda_{P}$ at $\lambda_{F}=$ const $=0.15$ (right). Dashed and dash-dotted lines in (b) indicate the position of the laser sheet during the measurements with the respect of each repeated unit for the $2 \mathrm{D}$ and $3 \mathrm{D}$ PIV setup respectively. Dimensions are not in scale for ease of readability. Flow is top to bottom in (b). 


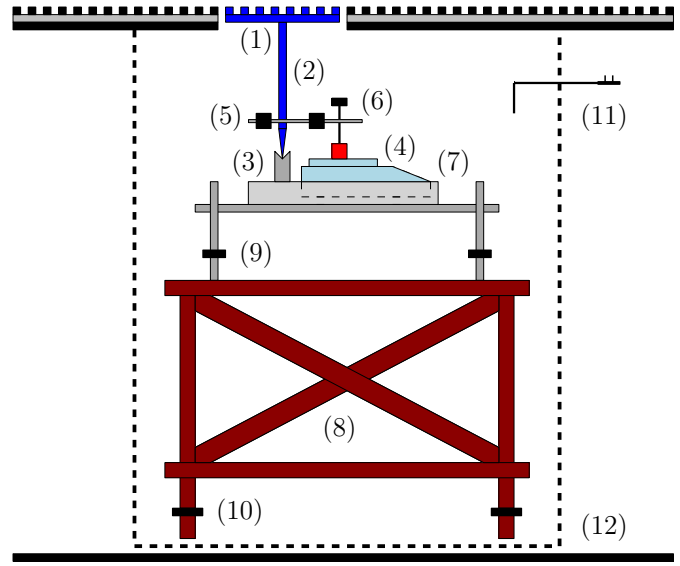

FIGURE 4. Schematic of the floating element drag balance. Components: (1) measurement patch, (2) two-arm lever system, (3) knife edge, (4) precision scale, (5) counterbalance weights, (6) loadtransfer adjustment pin, (7) H-frame containing the scale, (8) base structure, (9) fine adjustment feet, (10) rough adjustment feet, (11) Pitot tube, (12) sealed box.

planes were acquired at the spanwise centreline of the test section by a $16 \mathrm{M}$ pixel highresolution camera equipped with Nikon $105 \mathrm{~mm} \mathrm{f} / 8$ lenses at a fixed sampling frequency of $0.8 \mathrm{~Hz}$. This is so that statistically uncorrelated measurements are acquired for each image pair. For each run, 2000 image pairs were acquired and processed with DaVis 8.0 software. The setup allowed a field of view of $200 \mathrm{~mm} \times 136 \mathrm{~mm}$ (approximately $1.8 \delta \times 1.3 \delta$ streamwise-wall-normal for each roughness configurations). Velocity vectors were obtained using $16 \times 16$ pixel $^{2}$ final interrogation windows with $50 \%$ overlap. The resulting spatial resolution is approximately $0.7 \mathrm{~mm} \times 0.7 \mathrm{~mm}$ for all cases and successive vectors are spaced at half that distance (due to $50 \%$ overlap). Images were preprocessed with averaged minimum intensity background subtraction. After this procedure, a field of $97-99 \%$ good vectors was achieved, minimising the need of interpolation (when necessary a local mean interpolation was used).

The time delay between laser pulses across all the cases was chosen such that the pixel displacement in the freestream was approximately 15 pixels. This ensures that the bias error of the PIV velocity measurements is less than $1 \%$ of the full-scale velocity (given that the sub-pixel determination has an uncertainty of about \pm 0.1 pixels - see Adrian \& Westerweel 2011). The overall uncertainty in turbulence quantities was determined, following Benedict \& Gould (1996) and was found to be less than $1 \%$ in the mean velocity, $5 \%$ and $8 \%$ for the streamwise and normal turbulence intensities and $10 \%$ for the shear stresses. These values are also in line with previous PIV-based studies on rough-wall turbulent flows (e.g. Wu \& Christensen 2007).

The resolution of the current $2 \mathrm{D}$ data sets ranges between 28 to 42 wall-units, due to differences in the skin friction velocities generated by the different surface morphologies. The different local resolution has a tangible effect on the turbulence statistics, in particular on the higher-order quantities. Therefore, for all the 2D PIV results presented herein, the data sets have been filtered with a low-pass Gaussian filter designed to match the local resolution at $l_{2 D}^{+}=45$. It must be noted that this filtered spatial resolution is comparable (if not better) than previous cross-wire and PIV based measurements presented in the literature. 


\subsubsection{Stereoscopic PIV}

Given the high relative roughness height $(h / \delta)$, spanwise flow heterogeneity is to be expected within the roughness sublayer. To investigate this, stereoscopic PIV measurements were also carried out in wall-normal-spanwise $(y, z)$ plane at roughly the same location of the 2D measurements. Two cameras with the same specifications as for the 2D measurements were used to acquire 1500 digital image pairs. A FOV covering a minimum of two spanwise repeated-units $(1.5 \delta \times 1.5 \delta$ spanwise-wall-normal $)$ was resolved. A $1.5 \mathrm{~mm}$ thick laser sheet was used to illuminate the particles and the time delay between the laser pulses $(\Delta t=20 \mu s)$ had to be reduced accordingly so that the particles would not leave the laser sheet plane. This reduced $\Delta t$ has an influence on the averaged pixel displacement of the particles, and hence the PIV uncertainty. Measurements were carried out only across six cases: LF2, LF3, LF5 and LP2, LP4, LP5 as these represent conditions for sparse, medium and dense solidity regimes. The cameras were oriented at an angle of approximately $25^{\circ}$ to the measurement plane axis, which is appropriate to accurately resolve the out-of-plane velocity component (Raffel et al. 1998). Scheimpflug adaptors were mounted on the lenses to help maintain focus across the entire field of view by orienting the lens plane at an angle to the image plane, as discussed in Raffel et al. (1998). A two-plane calibration target was used for calibration, and self-calibration using Davis 8.0 was applied to improve the quality of the final velocity field (typically up to 2-pixel correction was used). Velocity vectors were obtained using $32 \times 32$ pixel $^{2}$ final interrogation windows with $50 \%$ overlap. This resolution is coarser than the planar PIV measurement, however, given the nature of measurements (i.e. flow perpendicular to laser sheet) and the thickness of laser sheet, this interrogation window is deemed sufficient. The worst case resolution $\left(l_{3 D}^{+}\right)$across the different cases was approximately 120 wall-units, therefore for the same reasons previously discussed, the 3D dataset has been filtered to match the local resolution across cases at $l_{3 D}^{+}=125$. This is comparable to similar measurements in the literature.

\subsection{Indirect estimate of skin-friction}

The skin friction velocity, $U_{\tau}=\sqrt{\tau_{w} / \rho}$, where $\tau_{w}$ is the wall total shear stress and $\rho$ is the density of the fluid, is commonly assumed to be the average Reynolds shear stress in the log-region. In this paper, we refer to this as an indirect method for determining skin-friction velocity. Cheng \& Castro (2002b) in their experiments on different urban roughnesses (all with $\lambda_{F} \equiv \lambda_{P}=0.25$ ) demonstrated that an optimum estimation for the skin friction velocity can be obtained from spatially averaged Reynolds shear stress within both the roughness sublayer (RSL) and the inertial sublayer (ISL). The reader is referred to $\S 3.2$ for further details on these regions. This shows that by spatially averaging over a roughness repeating unit, the logarithmic region can be extended to the roughness sublayer. In our investigation, the average across the streamwise direction and over a single repetitive unit yielded, within experimental uncertainty, the same results. Furthermore, Cheng et al. (2007) also argued that for boundary-layer flows over staggered arrays of cubical elements, the $\rho \overline{u v}$ underestimates the surface stress by some $25 \%$. Therefore, a corrected estimate should be used, defined as (Reynolds \& Castro 2008),

$$
U_{\tau}=\left.1.12 \sqrt{-\overline{u v}}\right|_{2<y / h<3} ;
$$

where the Reynolds shear stress is evaluated from the plateau region in the roughness sublayer (as in Flack et al. 2005 and Castro 2007). Both these above-mentioned approaches yield a $U_{\tau}$ value that is within $5 \%$ of each other. This value is also similar to 
the $U_{\tau}$ obtained by assuming it to be the maximum of the Reynolds shear stresses as in other studies in the literature (Manes et al. 2011).

\subsection{Determination of aerodynamic parameters}

the skin friction velocity is known (as measured via the drag-balance), a least-squarefit procedure was adopted to evaluate the zero-plane displacement, $d$, and the roughness length, $y_{0}$. This procedure is applied on the mean velocity field that was obtained by first ensemble averaging the data followed by streamwise averaging across the entire FOV. The log-layer is assumed to exist for $1.5 h \leqslant y \leqslant 0.2 \delta$ as in Schultz \& Flack (2005). The fitting procedures were carried out with $\kappa=0.38$ for all surfaces. This value is close to the value of $\kappa$ suggested by Marusic et al. (2013), for high Reynolds number smooth-wall boundary layers, where $\kappa=0.39 \pm 0.02$. A different choice of $\kappa$ alters the numerical values of $y_{0}$ and $d$ but not the trends shown in the following sections. It is important to highlight that, although is of common practice, fitting velocity profiles to determine the unknowns in equation 1.1, bares great uncertainty. This is due to the difficulties, particularly at the large $h / \delta$, in determining both the skin friction velocity and the log-law boundaries (Acharya et al. 1986; Castro 2007; Segalini et al. 2013). The reduction of the wall-normal range of the logarithmic layer, due to the increasing importance of the RSL is another important factor to take into account.

\section{Results and discussion}

Section 3.1 shows the effect of the surface morphology on the aerodynamic parameters. Section 3.2 examines the effect of the wall morphology on the roughness sub layer (RSL). Finally section 3.3 introduces the details of the proper orthogonal decomposition analysis and its implementation to examine the global spatial structure of the flow over different types of roughness. The turbulent kinetic energy content at different flow scales is also investigated.

\subsection{Effect of surface morphology on aerodynamic parameters}

Before examining the effect of the surface morphologies on aerodynamic parameters, it is useful to determine if these surfaces are fully-rough, in order to ensure that the results presented herein are solely due to the different roughness morphologies, rather than the product of a change in Reynolds number (i.e. viscous effects). There is general consensus in the literature that fully-rough conditions are attained when $y_{0}^{+}>2$ (Castro 2007 ) to $h_{s}^{+}>70$ (Flack et al. 2005). Here $h_{s}$ is the equivalent sand-grain roughness as in Nikuradse (1933). Recent findings have cast doubt on these limits and pointed out that, depending on the surface morphology, values up to $y_{0}^{+}>10$ might be necessary to guarantee the fully-rough conditions (Castro et al. 2013). In order to assess if the surfaces in the current study are in the fully-rough regime, the friction coefficient, $C_{f}=$ $2\left(U_{\tau} / U_{\infty}\right)^{2}$, was measured with the drag-balance at different inflow velocities and hence, different Reynolds numbers $\left(R e_{h}=U_{\infty} h / \nu\right)$. Velocity range in between 11.5 to $20 \mathrm{~m} / \mathrm{s}$ were considered and the results are presented in figure $5(a) \&(b)$ for the frontal and plan solidity cases respectively. It is clear that the variation in the measured friction coefficient at different velocities is within the limits of uncertainty. It follows that, within the measurement uncertainty, all cases in both $\lambda_{F}$ and $\lambda_{P}$ are to be considered in the fully-rough regime, i.e. Reynolds number independent. Hence, if differences are observed in the aerodynamic parameters, these are purely an effect of surface morphology. It should be pointed out that additional velocities were tested (down to $6 \mathrm{~m} / \mathrm{s}$ ). These results are here omitted as the measurement resolution was lower than the presented cases. However, 
10

(a)

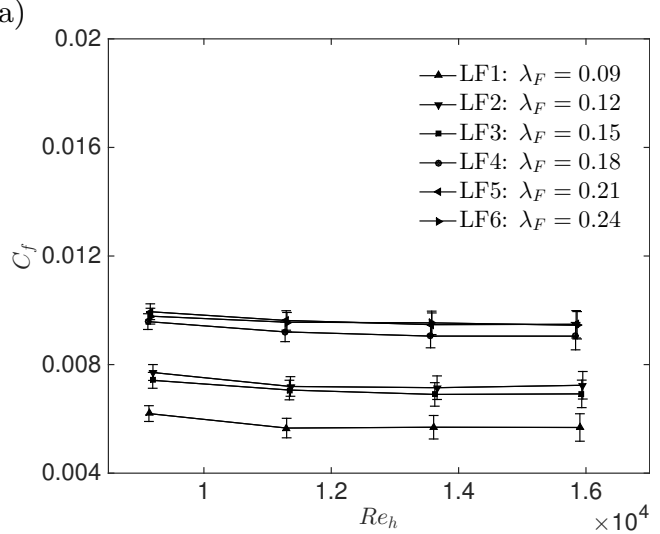

M. Placidi and B. Ganapathisubramani

(b)

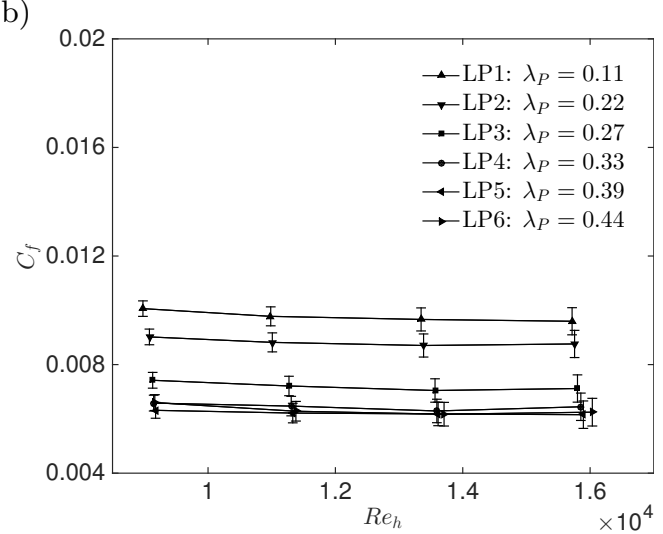

Figure 5. Skin friction coefficient, $C_{f}$, as a function of Reynolds number, as a function of $(a)$ $\lambda_{F}\left(\lambda_{P}=\right.$ const $\left.=0.27\right)$ and $(b) \lambda_{P}\left(\lambda_{F}=\right.$ const $\left.=0.15\right)$.

these clearly showed Reynolds number dependance of the skin friction (i.e. transitionally rough regimes). Increasing the Reynolds number, nevertheless, $C_{f}$ shows the plateaux described above, which is an indication that the "fully rough regime" is reached.

The validity of the Reynolds-stress method (via fitting of equation 2.1) for the skin friction determination can be established by comparing the results with the direct frictionbalance measurements. The value of $C_{f}$ as a function of the normalised momentum thickness, $\theta / y_{0}$, is shown in figure 6 . In particular, figure $6(\mathrm{a})$ shows results for the indirect method (via equation 2.1) and figure 6(b) using the direct method (friction-balance) for skin-friction estimation. This type of plot was first proposed by Castro (2007) and will allow us to estimate if the current data agrees with previous findings. Most of the results from rough-wall studies have been found to scatter around the two curves (for different values of wake parameter $\Pi$ ) shown in figure 6 (Castro 2007). It can be seen that our data using both methods are consistent with previous results in the literature. The indirect measurements results (via equation 2.1) compare well with the direct measurements (within 10\%) given the uncertainty in determination of the skin-friction velocity (Acharya et al. 1986) and the log-law boundaries used to determine the roughness length (Segalini et al. 2013). The skin friction values obtained via the floating-element dragbalance are used in all our analysis. Relevant boundary-layer characteristics of all the cases are given in table 1 . It can be seen from the table that all cases have $y_{0}^{+} \geqslant 10$ and hence we conform to the revised estimates for fully-rough conditions.

Figure $7(a)$ shows the mean velocity profiles in inner scales for the different cases of $\lambda_{F}$ at fixed $\lambda_{P}$. It can be seen that, compared to a smooth-wall case (equation 1.1 with $d=0$, $B=5$ and $\Delta U^{+}=0$ ), the roughness is responsible for a uniform downward shift of the log-region, as expected. The plain baseboard case, referring to the wind tunnel floor being covered only with baseboard but no bricks, is also reported for comparison. It shows that the presence of the blocks (case LF1 to LF6) is indeed responsible for generating a further shift of the log-law compared to the baseboard case. Figure $7(b)$ shows the behaviour of the mean velocity profiles for the different $\lambda_{P}$ cases. Once again the roughness is responsible for a downward shift of the log-law region. According to Jimenez (2004), the log-layer over a rough surface should remain intact only for $h / \delta<1 / 40$, so that the velocity profiles should be self-similar in the other region. However, when $h / \delta>1 / 40$, a situation similar to flows over obstacles is encountered and the universality of velocity statistics breaks down. A discussion on the critical relative roughness height, $h / \delta$, for which Townsend's similarity hypothesis fails is outside the scope of this study. However, 


\begin{tabular}{lcccccccccccc} 
Data set & $\lambda_{F}$ & $\lambda_{P}$ & $\delta^{*}(m m)$ & $\theta(m m)$ & $d(m m)$ & $U e(m / s)$ & $U_{\tau}(m / s)$ & $R e_{\tau}$ & $\delta / h$ & $\delta / h_{s}$ & $y_{0}{ }^{+}$ \\
\hline LF1 & 0.09 & 0.27 & 19 & 13 & 11.2 & 11.57 & 0.65 & 5110 & 10 & 69 & 10 \\
LF2 & 0.12 & 0.27 & 27 & 16 & 6.7 & 11.61 & 0.73 & 6313 & 11 & 31 & 28 \\
LF3 & 0.15 & 0.27 & 26 & 16 & 9.1 & 11.65 & 0.71 & 6140 & 11 & 36 & 24 \\
LF4 & 0.18 & 0.27 & 28 & 16 & 8.5 & 11.53 & 0.80 & 6919 & 11 & 16 & 61 \\
LF5 & 0.21 & 0.27 & 32 & 18 & 7.0 & 11.63 & 0.82 & 7092 & 11 & 13 & 77 \\
LF6 & 0.24 & 0.27 & 31 & 17 & 8.3 & 11.64 & 0.81 & 7005 & 11 & 14 & 69 \\
\hline LP1 & 0.15 & 0.11 & 35 & 19 & 5.7 & 11.54 & 0.81 & 7642 & 12 & 12 & 89 \\
LP2 & 0.15 & 0.22 & 30 & 17 & 7.7 & 11.57 & 0.78 & 6746 & 11 & 16 & 60 \\
LP3 & 0.15 & 0.27 & 28 & 16 & 11.4 & 11.58 & 0.71 & 6140 & 11 & 30 & 29 \\
LP4 & 0.15 & 0.33 & 27 & 16 & 10.4 & 11.50 & 0.67 & 5794 & 11 & 31 & 26 \\
LP5 & 0.15 & 0.39 & 24 & 15 & 11.1 & 11.49 & 0.66 & 5189 & 10 & 39 & 18 \\
LP6 & 0.15 & 0.44 & 21 & 13 & 11.1 & 11.42 & 0.67 & 5268 & 10 & 54 & 14
\end{tabular}

TABLE 1. Relevant experimental parameters for frontal and plan solidities variation. $U_{\tau}$ values reported are obtained via floating-element drag-balance. This value of $U_{\tau}$ is used in all subsequent analysis. The aerodynamic parameters are calculated through a log-law fit with $\kappa=0.38$ in the range $1.5 h \leqslant y \leqslant 0.2 \delta$. The $U_{e}$ values reported herein are from the PIV results.

(a)

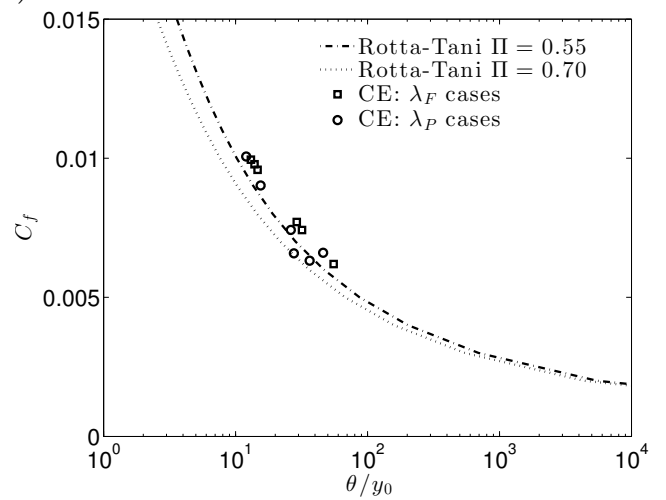

(b)

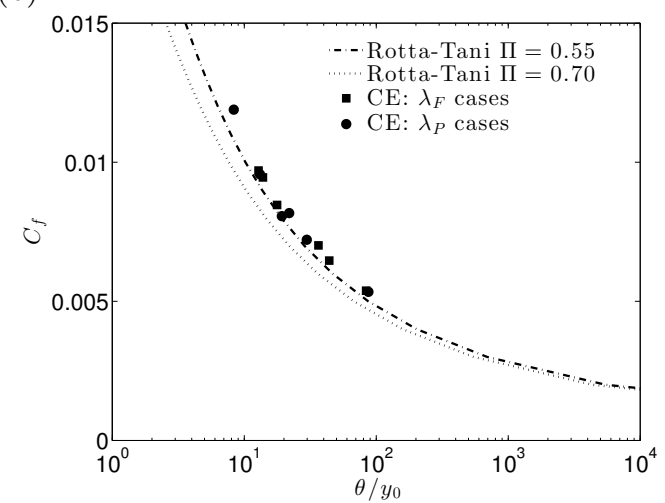

FiguRE 6. Variation of $C_{f}$ as a function of $\theta / y_{0}$ for (a) Reynolds-stress method (via fitting of equation 2.1) and (b) drag-balance method (direct measurement). Dot and dashed-dotted curves refer to the standard two-parameter family results with wake strength of $\Pi=0.55$ and 0.7. The roughness length, $y_{0}$, is calculated via a least-square-fit procedure for $1.5 h<y<0.2 \delta$.

it can be clearly observed in figure 7 that a log-law seems to be well-defined for all velocity profiles. This is not unique given that previous studies (Castro 2007; Amir \& Castro 2011, amongst others) have shown that some rough-walls conform to outer-layer similarity up to $h / \delta \approx 0.2$, which is even more severe than the morphologies presented herein. It is therefore anticipated that Jimenez's criterion seems to be unnecessarily restrictive, given that these surfaces show mean velocity similarity.

To further quantify the effect of the surface morphology on the bulk drag, the roughness function can be calculated. Figure 8 shows the normalised roughness length, $y_{0} / h$, as a function of both solidities. It is shown in figures $8(a) \&(b)$ that the behaviour of the roughness length as a function of frontal and plan solidity is drastically different. Figure $8(a)$ shows that the roughness length (which is related to the total drag) increases in the sparse regime, and indicates a marginal decrease after the peak for increased values of 
(a)

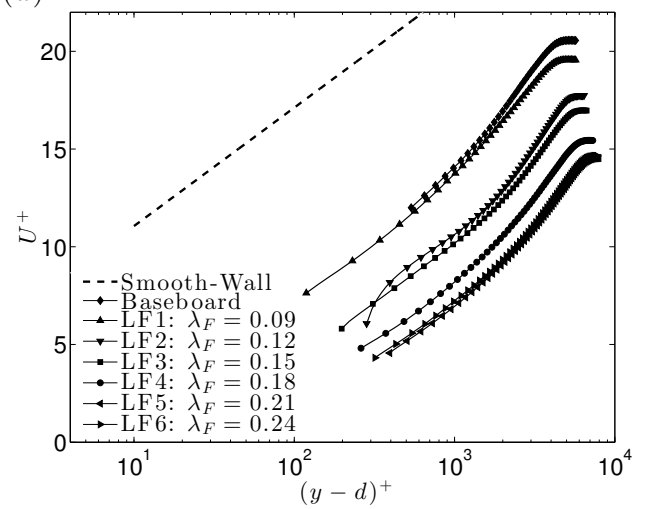

M. Placidi and B. Ganapathisubramani

(b)

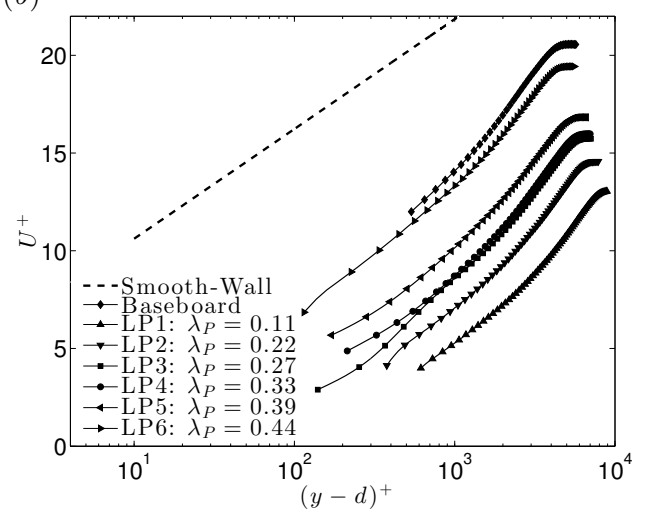

FIGURE 7. Mean velocity profiles in inner scales as a function of $(a) \lambda_{F}\left(\lambda_{P}=\right.$ const $\left.=0.27\right)$ and $(b) \lambda_{P}\left(\lambda_{F}=\right.$ const $\left.=0.15\right)$. Solid lines represent the real data resolution whist markers are spaced every five vectors for clarity.

frontal solidity, as in Leonardi et al. (2003) and Leonardi \& Castro (2010). Within the range of solidity explored herewith, the bulk drag seems to reach a peak at $\lambda_{F}=0.21$ for a fixed $\lambda_{P}=0.27$. The roughness length results, in figure 8(a), closely follow the skin friction trends as a function of both the solidities, as shown in table 1. The drag-peak location herein is partially in contrast with previous studies, for which it was found at $\lambda_{P} \equiv \lambda_{F} \approx 0.15$ (Hagishima et al. 2009; Leonardi \& Castro 2010; Kanda et al. 2004) and $\lambda_{P} \equiv \lambda_{F} \approx 0.16$ (Santiago et al. 2008; Coceal \& Belcher 2004). These differences are perhaps not surprising giving the high uncertainty in the fitting procedure which results in the visible scatter of the data for different studies in figure $8(a)$, even when values of similar frontal and plan solidity are considered. This scatter could also be partly due to the fact that prescribing a set of $\lambda_{F}$ and $\lambda_{P}$ values, results in a non unique mapping of a roughness geometry - i.e. different geometries can generate the same combination of solidities. Furthermore all the previous studies used cubical elements, for which $\lambda_{F} \equiv \lambda_{P}$ while in the current experiment the plan solidity has been kept constant. Additionally, the comparable studies also have different $h / \delta$ values. Finally, given that the current dataset is a collection of discrete points, the reported peak at $\lambda_{F}=0.21$ only implies that the effective peak value should be located somewhere in the range $\lambda_{F}=0.18-0.24$ which is consistent with previous findings. It is important to note that this result only apply within $0.11 \leqslant \lambda_{F} \leqslant 0.27$, as no information is available outside this range.

It must be highlighted that the existence of this peak in the bulk drag is not necessarily in agreement with Macdonald's prediction, which prescribes a monotonic increase of the drag as the element's frontal density increases. Given the scatter in data, it is difficult to interpret this further. However, as emphasised previously, in the current experiment the influence of frontal and plan solidity have been decoupled keeping one of the two parameters fixed at a time and trend does suggest a peak in drag for $\lambda_{F}$ at a given $\lambda_{P}$. The behaviour of the bulk drag with plan solidity is shown in figure $8(b)$ and it reveals a completely different feature. The roughness length is found to monotonically decrease, as the plan density increases. It is not possible to compare this behaviour with previous studies for which $\lambda_{F} \equiv \lambda_{P}$, since in that case changes in one solidity parameter result in modifying the other one.

It is expected that the roughness length should increase as the elements' frontal density increases, reaching a peak beyond which it should decrease, for further increase in roughness density. This expected behaviour is verified in the case of frontal density variation, 
(a)

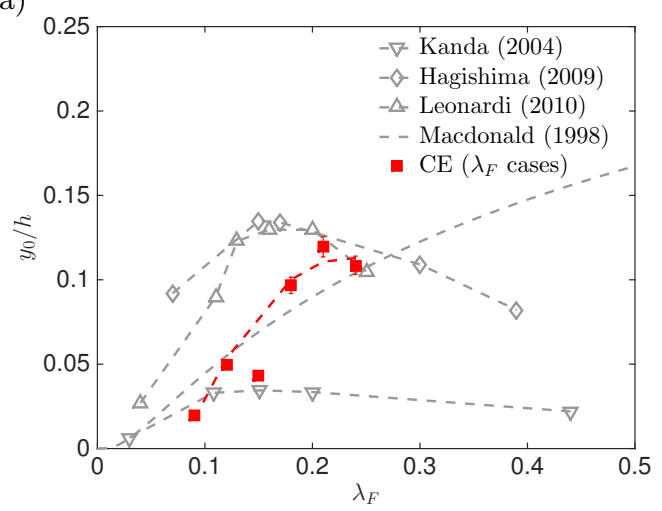

(b)

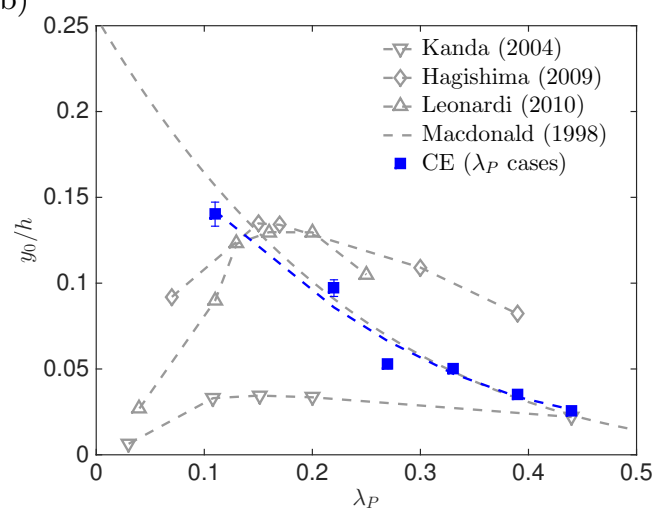

FiguRE 8. Normalised roughness length, $y_{0} / h$, as a function of $(a) \lambda_{F}$ and $(b) \lambda_{P}$. Current experiment refers to values obtained by log-law least-square fit between $1.5 h<y<0.2 \delta$.

which shows a peak for $\lambda_{F}=0.21$. An increase in frontal solidity (cases LF1 to LF6) increases the frontal blockage, reducing the free-space available for the flow, forcing it up and over the elements, and hence resulting in a drag increase. However, for plan solidity cases, the bulk drag monotonically decreases with increasing $\lambda_{P}$. This could be due to the fact that progressive increase of the plan area at fixed $\lambda_{F}$, and hence at fixed unit wall-parallel area (as in cases LP1 to LP6) results in a transition from "k-type" to "dtype" roughness. This progressive transition results in a decrease in drag with increasing plan solidity. It must be noted that we use the "k-type" or "d-type" terms only to relate to the flow mechanisms that are shown to be associated with these classifications and not to the type of roughness. The flow behaviour between the roughness elements, following Grimmond \& Oke (1999), depends upon the ratio between the roughness height, $h$, and the average streamwise spacing between elements, $W_{c}$ (the width of the channel between subsequent elements). An "isolated flow regime" is expected when $h / W_{c}<0.3$, a "wake interference regime" for $0.3<h / W_{c}<0.65$ and a "skimming flow regime" for $h / W_{c}>0.65$. It is easy to verify from figure $3(b)$ that, while the average spacing is almost constant for the $\lambda_{F}$ variation, the same is not true for the $\lambda_{P}$ cases. In the latter, in fact, this spacing decreases from case LP1 to LP6. This is because of the necessity to keep the unit wall-parallel area fixed, enforcing changes in plan solidity at fixed $\lambda_{F}$. Therefore, this results in cases LF1 to LF6 all belonging to the "wake interference regime" $\left(h / W_{c} \approx 0.39\right)$, while a transition between the "isolated flow regime" toward the "skimming regime" is to be expected between cases LP1 and LP6 $\left(h / W_{c}\right.$ ranging between $0.27-0.55)$.

It is certainly possible under some circumstances that an increase in frontal blockage can be in competition with the transition from "k-type" to "d-type" (i.e. changes in $\left.h / W_{c}\right)$. In those cases, the bulk drag of different surfaces with different $\lambda_{F}$ and $\lambda_{P}$ can be a constant. Alternately, depending on the pattern of the repeating unit, the drag could even decrease with increasing $\lambda_{P}$. A sketch that describes the scenarios discussed above is shown in figures 10 and 11 respectively. Figures 10(a) to $(c)$ show a progressive increase of the spanwise blockage in the flow. This results in the flow raising upward toward the top of the elements and over them (i.e. "k-type") resulting in an increase in bulk drag. Figure $11(d)$ to $(f)$ shows a representation of the transition mechanism with elements in subsequent repeated units becoming closer to each other, resulting in the formation of stable vortices in the grooves between the elements. This ensures that the eddy shedding from the elements into the flow, is progressively more negligible. These 
14

(a)

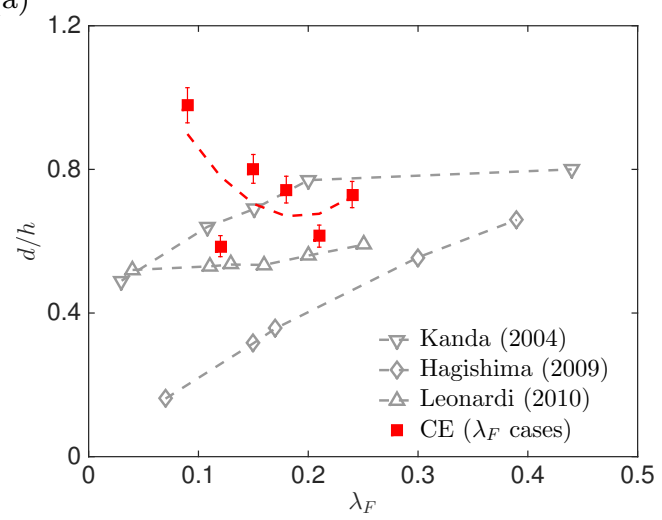

M. Placidi and B. Ganapathisubramani

(b)

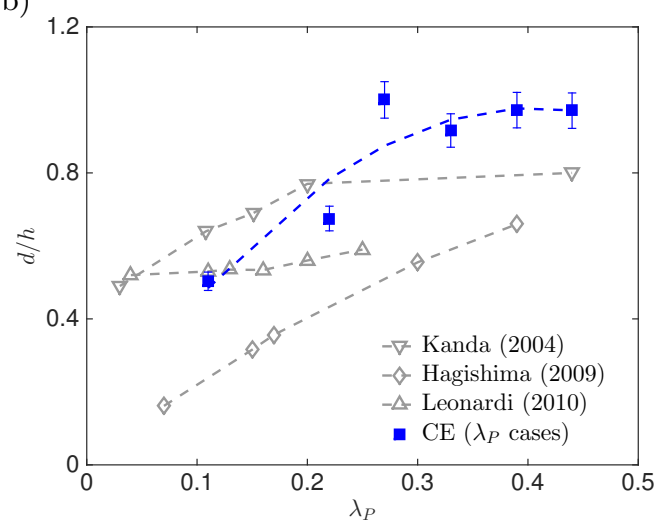

Figure 9. Normalised zero-plane displacement, $d / h$, as a function of $(a) \lambda_{F}$ and $(b) \lambda_{P}$. Current experiment refers to values obtained by log-law least square fit between $1.5 h<y<0.2 \delta$.

stable recirculation vortices are effectively isolating the elements from the flow (i.e. "dtype"), which rides almost undisturbed over the elements resulting in a reduction of the generated drag.

In addition to the roughness length, the values of the zero-plane displacement, $d$, as a function of frontal and plan solidities have also been calculated and are shown in figures 14(a) \& (b) for frontal and plan solidities, respectively. Since a value of $d>h$ would be physically meaningless (Jackson 1976), these values are forced to be $d=h$ as in Iyengar \& Farell (2001). It is clearly visible in figure 14(b) that the virtual origin increases with an increase in plan solidity till it approaches its asymptote at $d=h$. The latter is qualitatively consistent with previous studies which have shown that the zero plane displacement tends to assume larger values (i.e. $d$ tends to $h$ ) as the plan solidity increases (Kanda et al. 2004; Hagishima et al. 2009; Leonardi \& Castro 2010). This trend is also consistent with the predictions from Macdonald (1998). Although the quality of the fit deteriorates, an opposite trend seems to emerge in figure 14(a). For a frontal solidity variation, the virtual origin decreases as $\lambda_{F}$ increases. It must be noted that the trend followed by previous studies does not capture the conditions of the present experiments - the data was obtained over cube roughness and hence the behaviour with $\lambda_{F}$ is exactly the same as for $\lambda_{P}$.

\subsection{Effect of surface morphology on the roughness sublayer}

Given the high relative roughness $(h / \delta \approx 0.1)$, flow heterogeneity along the spanwise direction are to be expected at least within the roughness sublayer. This region has usually been identified to extend up to five roughness heights (Cheng \& Castro 2002a; Flack et al. 2007). To investigate the flow heterogeneity, results from stereoscopic PIV measurements in the wall-normal-spanwise $(y, z)$ plane are presented. Contour plots of mean streamwise velocity are shown in figure 12 for both $\lambda_{F}$ and $\lambda_{P}$ cases on the left and right column, respectively. The extent of the field of view has been cropped so that two to three entire repeated units are contained in the presented graphs, hence allowing the effect of the edges of the single unit to be taken into account. To facilitate the interpretation of the figures, the bricks locations are also highlighted in the figure. Three/four bricks combined represent one repeated unit for the $\lambda_{F}$ cases while only two bricks combine to form the $\lambda_{P}$ cases (as in figure $3(b)$ ) keeping the overall size of the FOV roughly the same. Full black bricks stand for elements in the measurement plane whilst dashed lines 
(a)

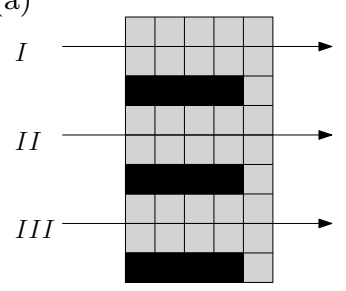

(d)

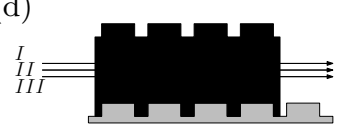

(b)

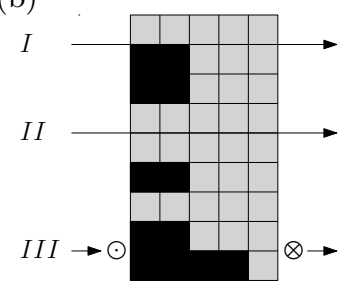

(e)

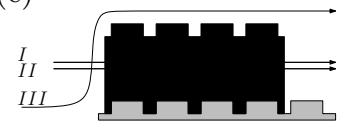

(c)

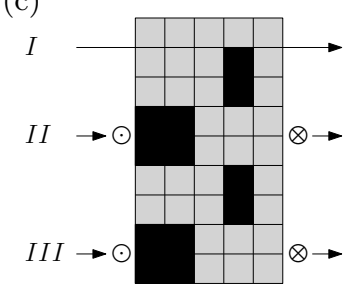

(f)

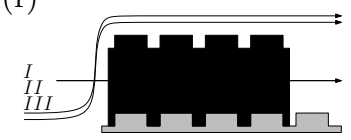

FiguRE 10. Different elements' field patterns and associated flow regimes for increasing $\lambda_{P}$. LF1 (left), LF3 (centre) and LF5 (right). Symbols $\odot$ stand for arrows pointing toward the reader and symbolise path lines of particles that deviate upward, symbols $\otimes$ stand for path lines of particles that deviate downward. Flow is left to right.

(a)

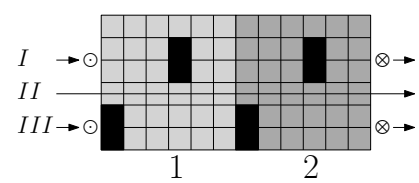

(d)

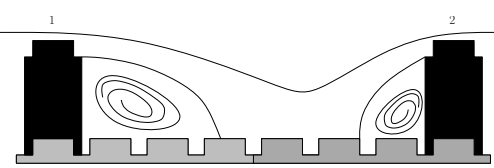

(b)

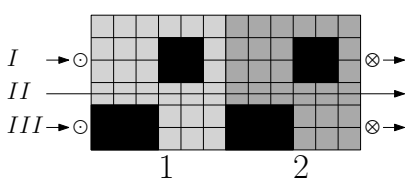

(e) (c)

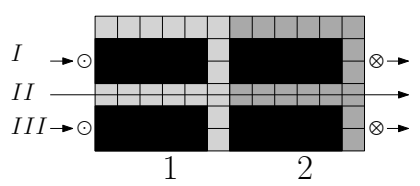

(f)

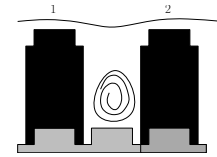

FiguRE 11. Different elements' field patterns and associated flow regimes for increasing $\lambda_{P}$. LP1 (left), LP3 (centre) and LP6 (right). Symbols $\odot$ stand for arrows pointing toward the reader and symbolise path lines of particles that deviate upward, symbols $\otimes$ stand for path lines of particles that deviate downward. Flow is left to right. Light and dark grey areas in $(a),(b)$ and (c) indicate subsequent elements' repetitive units.

represent bricks out of the measurement plane, yet in the same repeated unit. For the frontal solidity cases (in figure 12 on the left), a mean flow distortion due to the elements' field for low values of $y / h$ is clearly visible. This effect is much greater for the $\lambda_{F}$ cases than for the $\lambda_{P}$ cases (in figure 12 on the right). For all cases, the contour lines appear reasonably flat farther away from crest of the roughness elements suggesting a localised effect of roughness. Moreover very little differences in mean streamwise velocity across the span are found above $5 h$ height, which is therefore suggested to be the upper edge of the roughness sublayer.

There is very little information available in the literature on the definitions used to determine the depths of the inertial sublayer (ISL) and roughness sublayer (RSL), and this is subject of on-going debate (Cheng \& Castro 2002b,a). However, there is some consensus on the necessity to look at the Reynolds shear stress characteristics. It is outside the scope of this paper to further investigate the best way to define these regions and therefore definitions from previous literature are followed (Raupach et al. 1991; Cheng et al. 2007; Cheng \& Castro 2002b). The upper boundary of the RSL is defined as the location where the spatially-spanwise-averaged wall-normal profiles converge to within $10 \%$. Similarly, the ISL is defined as the region where the vertical variation of the 
(a)

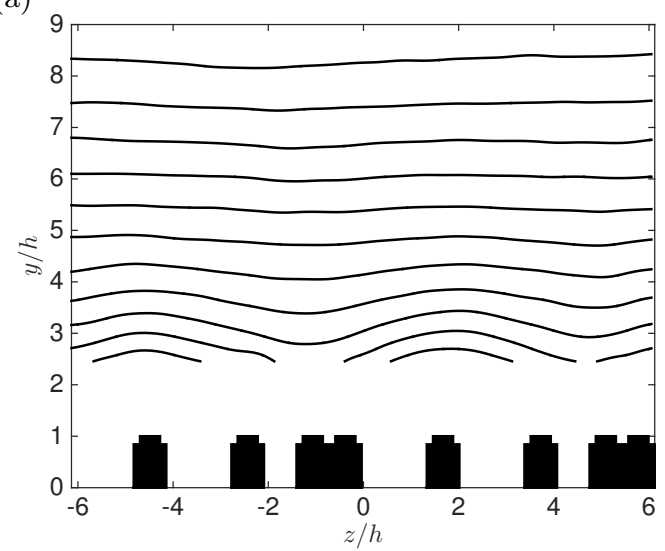

(c)

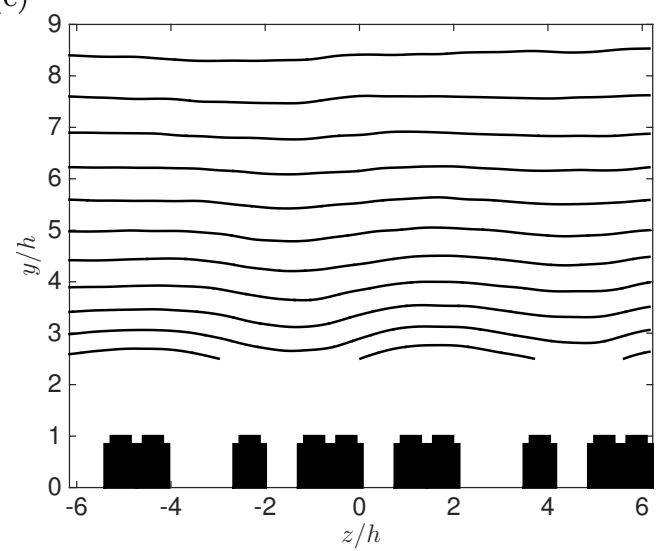

(e)

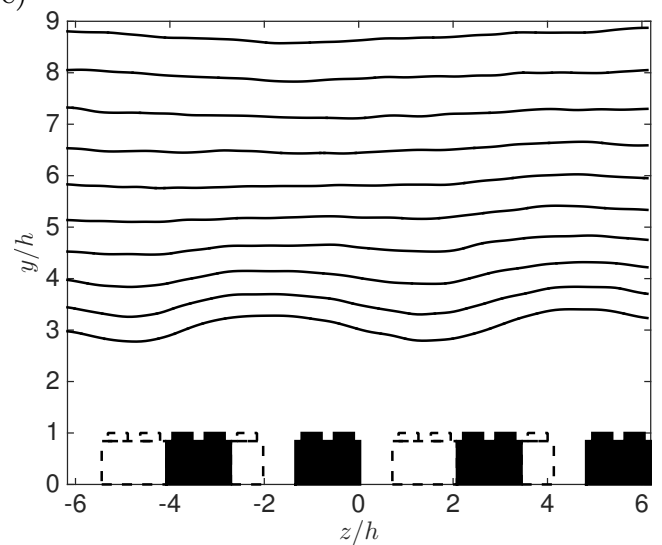

(b)

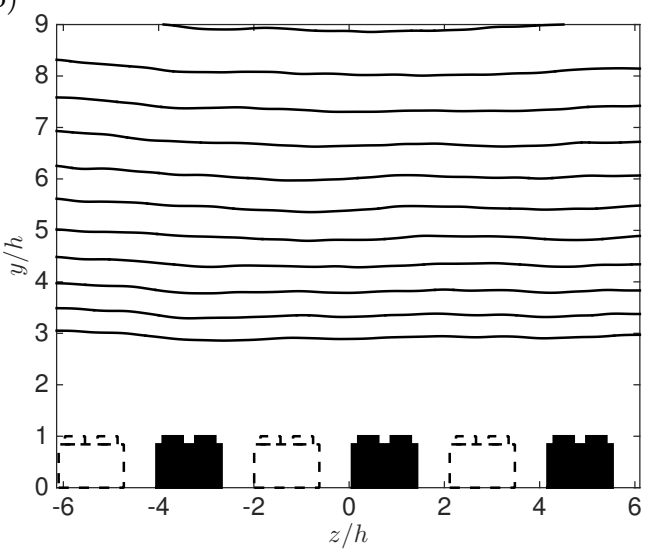

(d)

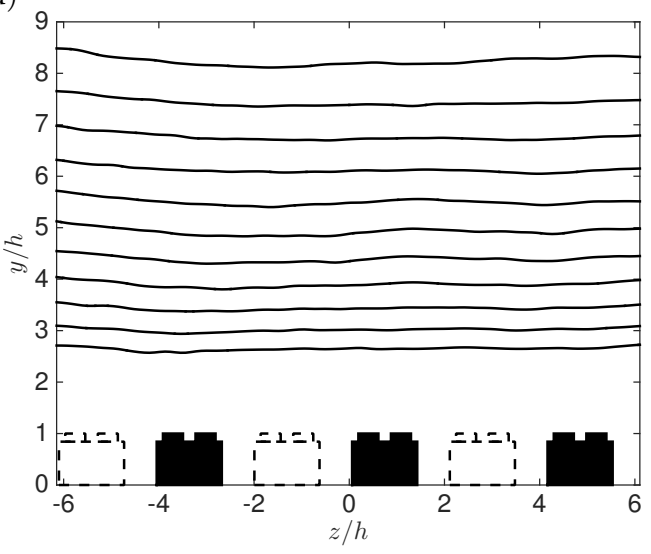

(f)

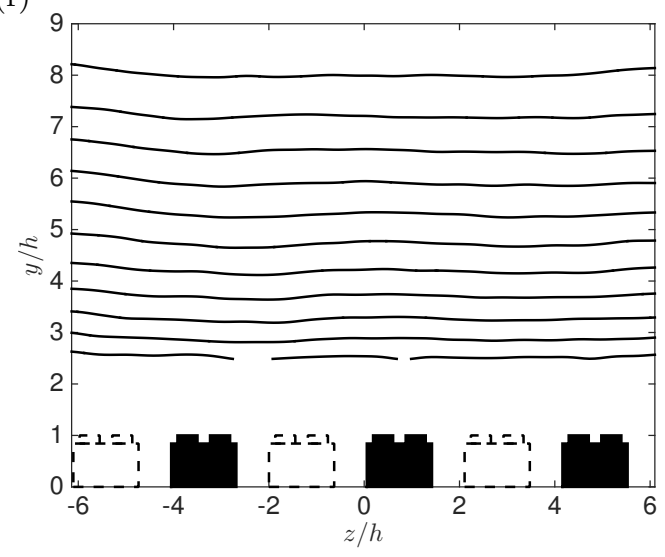

FiguRE 12. Mean (ensemble-averaged) streamwise velocity contours, $U$, in the $(y, z)$ plane for sparse (top), medium (centre) and dense regimes (bottom) as a function of $\lambda_{F}$ $\left(\lambda_{P}=\right.$ const $\left.=0.27\right)$ on the left and $\lambda_{P}\left(\lambda_{F}=\right.$ const $\left.=0.15\right)$ on the right. Lowest contour level $U / U_{e}=0.5$, contour spacing of 0.05 . Bricks in the measurement plane are represented in full black, whilst bricks out of the measurement plane in the same repeated unit are identified by dashed lines. The mean flow is in to the plane along positive $x$ axis. 
shear stress may be neglected or its variation is within a certain threshold (i.e. $<5 \%$ ). Both these regions are defined relative to the mean (ensemble) Reynolds shear stresses spatially-averaged across the FOV.

Figure 13 shows the extent of the above-mentioned regions within the boundary layer for frontal and plan solidity variation on the left and right column, respectively. Grey solid marks indicate profiles along the spanwise direction across the entire FOV, which included more than one complete repeated unit. In all these graphs, the roughness height is defined from the bottom of the legoboard (i.e. the actual wall $y=0$ ). The height of the roughness elements is marked by a dashed black line and it is constant. For all cases, the extent of the RSL is found to be confined to $\approx 5 h$ which is consistent with previous findings (Flack et al. 2007). Moreover, for the frontal solidity cases $((a),(c)$ and $(e))$ the RSL depth seems to reach a minimum to then increase again as the $\lambda_{F}$ increases. Therefore, the extent of this region follows a reverse trend when compared to that of the roughness length (or bulk drag) behaviour (in figure $8(a)$ ). It seems that an increase in drag results in a decrease of the RSL depth and vice-versa. This same trend is found for the plan solidity cases $((b),(d)$ and $(f))$ where the extent of the RSL seems to increase with the solidity, whilst the drag was found to decrease (see figure $8(b)$ ). The above-mentioned observations are summarised in figures $14(a) \&(b)$ that show the variation of RSL and ISL as a function of $\lambda_{F}$ and $\lambda_{P}$, respectively. It is important to note that differences can arise depending on the choice of threshold used to define the regions, however, the trend across all cases was found to be unaffected by the magnitude of the threshold.

It is worth noting that recent studies (Mejia-Alvarez \& Christensen 2013; Barros \& Christensen 2014) have experimentally shown that mean flow heterogeneities exist in the spanwise-wall-normal plane of rough-wall turbulent boundary layers, particularly over a complex roughness which exhibited large-scale streamwise-elongated patches of elevated height. This heterogeneity extend outside the RSL all the way to the freestream. This phenomenon is due to the presence of spanwise-wall-normal mean secondary flow in the form of mean streamwise vorticity associated with counter-rotating boundary layer-scale circulations (Barros \& Christensen 2014). The latter induces regions of high and low momentum (HMPs and LMPs respectively). Such patterns are also in agreement with the behaviour found by Reynolds et al. (2007) for flow over regular cubical roughness. The mean-flow heterogeneity in both cases were here found to be in correspondence of the periodic spanwise roughness spacing. However, although some of the cases herein in examination resemble Barros \& Christensen (2014)'s geometry (in particular case LP6), the current investigation found no trace of this heterogeneity in the freestream. This is in disagreement with the aforementioned studies and it is perhaps due to the fact that the spanwise spacing between elements in the cases examined in the current study is not significant enough to induce this characteristic behaviour. Further exploration of this aspect is outside the scope of this work.

\subsection{Proper orthogonal decomposition}

To explore the spatial characteristics and the behaviour of the flow and its dependence on wall morphology, a snapshot based proper orthogonal decomposition (POD) analysis has been carried out (Berkooz et al. 1993). This technique generates a basis for modal decomposition of ensemble of instantaneous fluctuating velocity fields and provides the most efficient way of identifying the motions which, on average, contain a majority of the turbulent kinetic energy (TKE) in the flow. The energy contribution of the singular value across the modes, hence its shape, depends on the local spatial resolution of the data set, as discussed in Pearson et al. (2013). This is because the energy content of each 
18

(a)

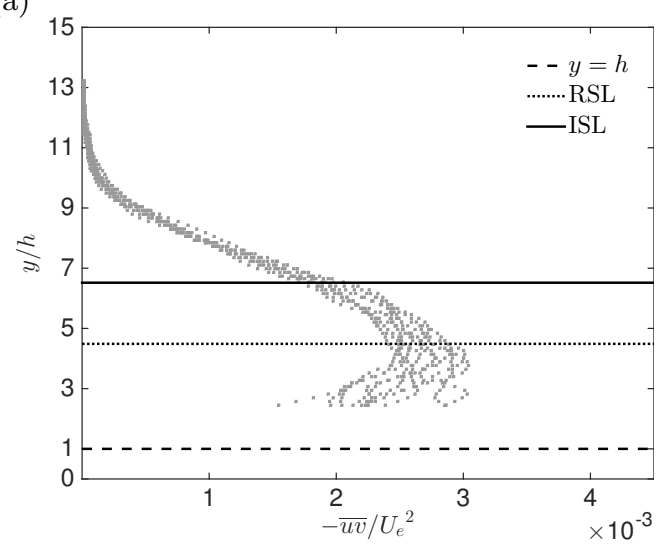

(c)

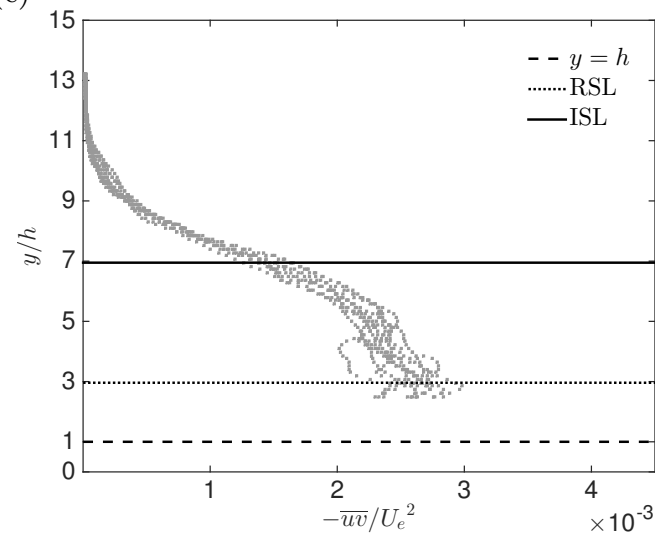

(e)

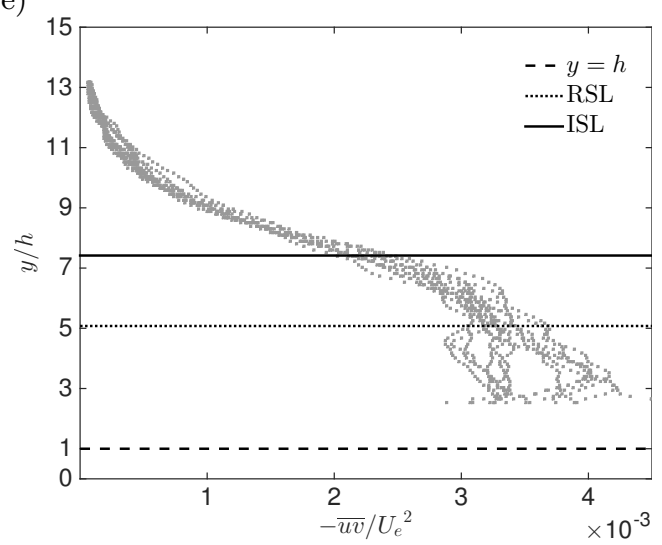

(b)

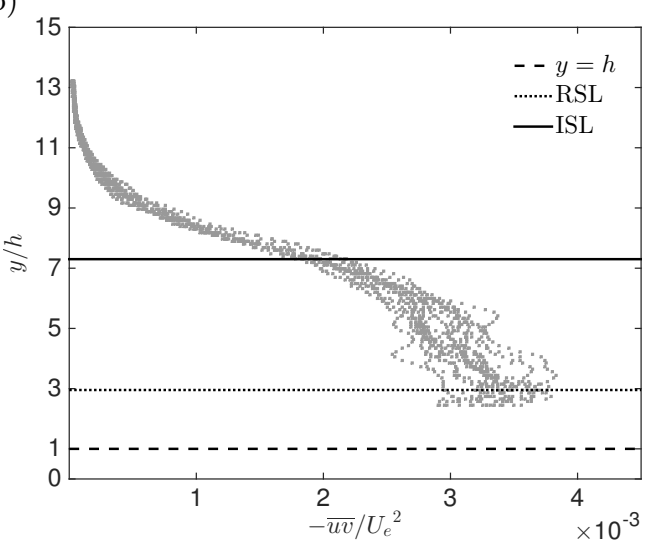

(d)

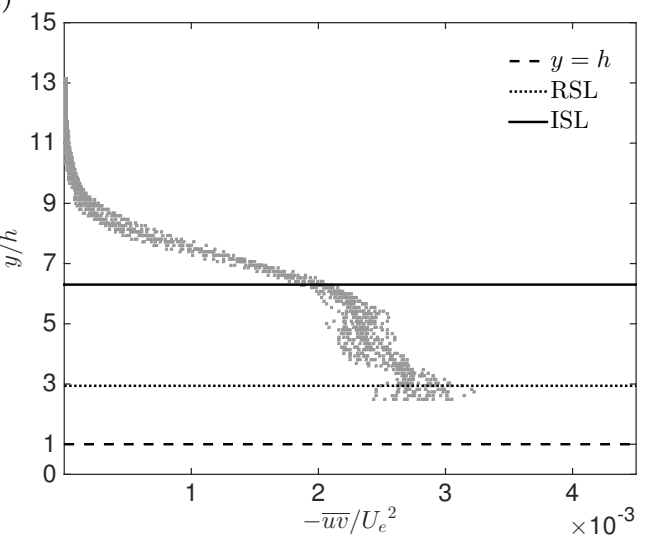

(f)

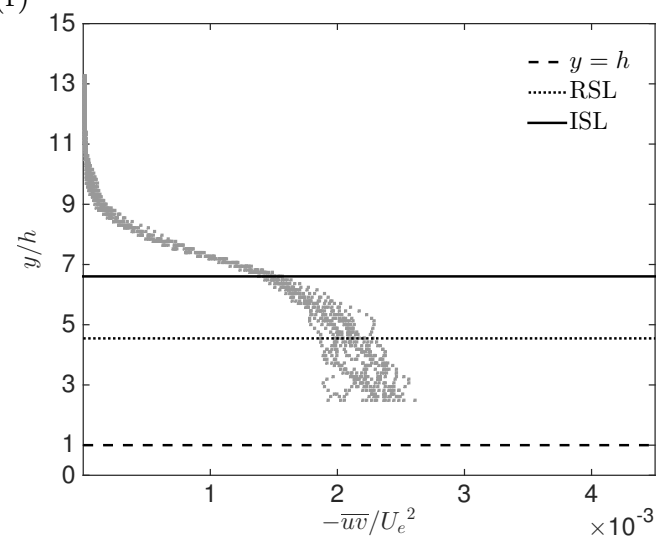

Figure 13. Normalised Reynolds shear stress profiles, $-\overline{u v} / U_{e}^{2}$, for sparse (top), medium (centre) and dense regimes (bottom) as a function of $\lambda_{F}\left(\lambda_{P}=\right.$ const $\left.=0.27\right)$ on the left and $\lambda_{P}$ $\left(\lambda_{F}=\right.$ const $\left.=0.15\right)$ on the right. Grey solid symbols represents the shear stress data (only one in every five vectors is shown for clarity), dashed black line shows $y=h$ (canopy layer) whilst dotted and solid black lines represent the extent of the RSL and ISL respectively. 
(a)

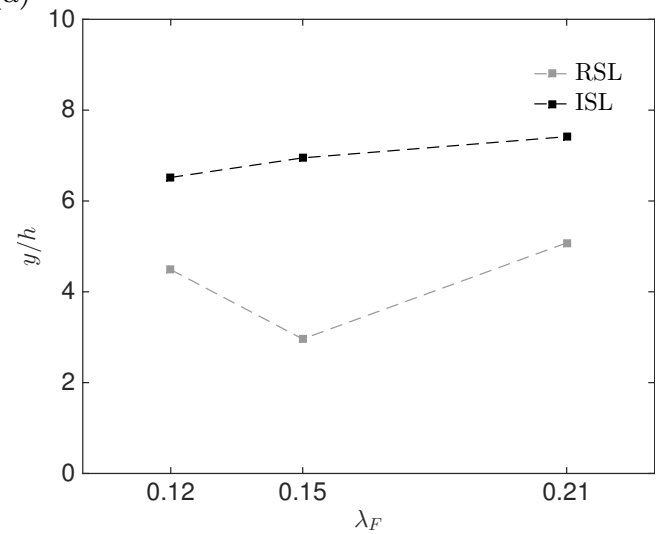

(b)

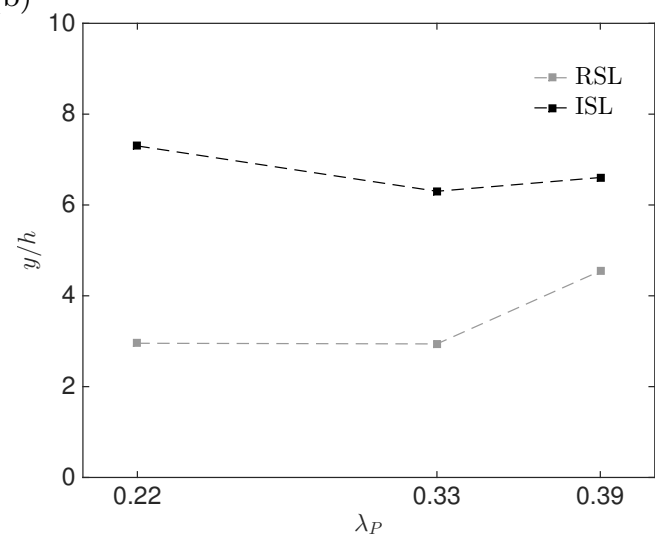

FIGURE 14. RSL and ISL depths as a function of $(a) \lambda_{F}\left(\lambda_{P}=\right.$ const $\left.=0.27\right)$ and $(b) \lambda_{P}$ $\left(\lambda_{F}=\right.$ const $\left.=0.15\right)$. Only available $3 \mathrm{D}$ data sets are presented.

$i$ th mode depends on the smallest resolved scale in the flow (i.e. in the data set). The global resolution of the current 2D data set ranges in between 30 to 40 wall-units, due to differences in the skin friction velocity generated by the different surface morphologies. This results in a variation of the Kármán number in the range of $R e_{\tau} \approx 4900-7500$. For this reason, the current data set has been filtered with a low-pass Gaussian filter designed to match the local resolution at $l_{2 D}^{+}=45$. Moreover, the FOV across the different cases is also matched to the region $-0.6 \delta<x<0.6 \delta$ in the streamwise and $1.5 h \leqslant y \leqslant \delta$ in the wall-normal direction to allow for meaningful comparisons. The result presented herein were obtained performing the POD calculation over the combined $(u, v)$ data.

Figure 15 shows contour plots of the shape of the first four energetic POD modes. These are specific to one case (i.e. LF2, $\lambda_{F}=0.09, \lambda_{P}=0.27$ ), although were found to be consistent (and indistinguishable) in shape and order across all surface morphologies, regardless of the regime. For comparison the first four modes for $\operatorname{LP} 2\left(\lambda_{F}=0.15\right.$, $\left.\lambda_{P}=0.11\right)$ are shown in figure 16 . The rest of the results are omitted for the sake of brevity. This similarity is remarkable considering the different patterns adopted to generate the morphologies (see figure 3(b)) and is a testimony of some form of universality across rough-walls. Regions of high and low streamwise momentum (or vice-versa), which are highlighted in black and white respectively are clearly visible in figure 15 (although the colorbar is arbitrary). The shape of the most energetic mode (mode 1) is characterised by an elongated large-scale high (or low) momentum region. Mode 2 embodies instead an inclined shear layer that separates a high-momentum region below it from a low-momentum region appearing above it (or vice-versa since the sign of the values is arbitrary and will depend on the eigenvalue itself). Similar shape is assumed by mode 3 where the inclination of the shear layer is reversed compared to the flow direction. Mode 4 is dominated by three distinctive regions: two localised low-momentum regions are separated by an elongated forward-leaning streamwise high-momentum region. As Adrian et al. (2000) discussed, while POD modes are not representative of the actual coherent structures present in the flow, but more of the energy of those structures, they do provide a qualitative glimpse of the dominant flow field associated with each mode and its variability from one mode to another. A trend of increasingly smaller structures for higher modes is generally found throughout all cases. Therefore, the low-order modes tend to be associated with large-scale structure, whilst the higher-order modes are representative of increasingly smaller scales. Comparison of the first ten modes across all 
20

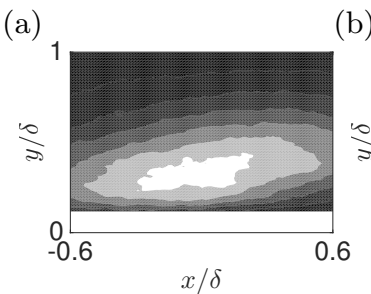

(b)

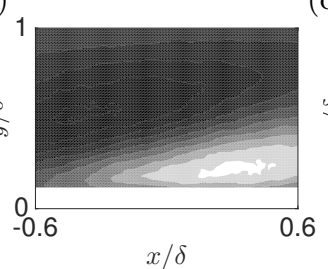

(c)

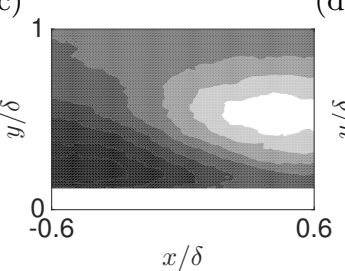

(d)

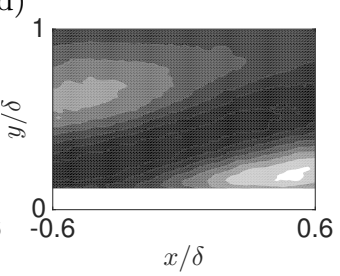

FiguRE 15. First four low-order POD modes for LF2 case. Flow is left to right. POD modes are calculated on the combined $(u, v)$ field.
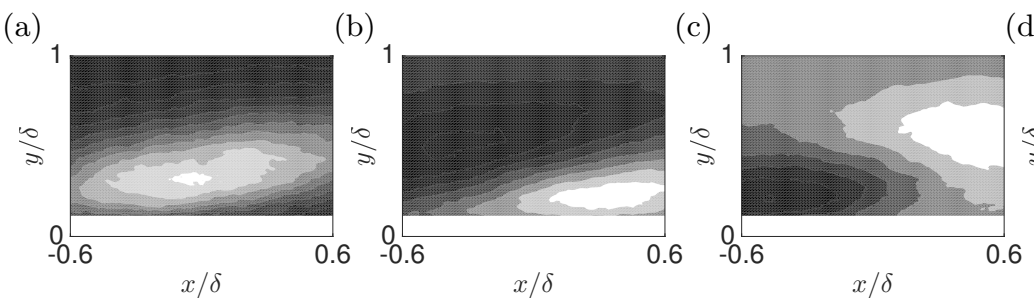

(d)

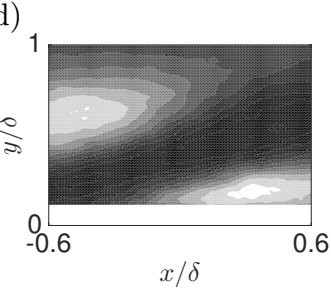

Figure 16. First four low-order POD modes for LP2 case. Flow is left to right. POD modes are calculated on the combined $(u, v)$ field.

the morphologies show identical mode shapes indicating that there is a degree of structural similarity regardless of roughness morphology (here omitted for brevity). Yet, the aerodynamic parameters are very different across the cases.

The differences in flow structure that perhaps lead to the differences in the aerodynamic parameters might not be evident when the structure of the entire boundary layer is examined using analysis of POD modes. It might be more meaningful to employ this analysis confined to the roughness sublayer so as to isolate any difference in the flow structures between the different roughness morphologies.

\subsubsection{Effect of surface morphology of the structure of the RSL}

To further investigate the effect of the surface morphology in the near-canopy (or nearwall) layer, the same POD analysis can be carried but this time only focussing on the roughness sublayer. As shown in the previous sections, this region appears to be where the roughness effect is confined. For this purpose, the POD analysis was carried out, as in the previous case, but the field-of-view was restricted to the roughness sublayer: $-0.6 \delta<x<0.6 \delta$ in the streamwise and $1.5 h<y<5 h$ in the wall-normal direction. The chosen vertical limit is based on the consensus in literature as the extent of the roughness sublayer $(y / h \approx 3-5$ as in Flack et al. (2007)). Figure 17 shows the result of this procedure for the frontal solidity cases. The top row, $(a)$ to $(d)$, shows results for the sparse regime (i.e. LF2) while the bottom row, (e) to (h), shows similar plots in the dense regime (i.e. LF5). The mode shapes appear to be qualitatively the same in this "near-canopy" fields, however, it must be highlighted that, for ease of readability, the y-axis is "stretched" compared to previous cases in figures 15 and 16 . The only difference between the two regimes (i.e. sparse vs. dense) is that modes 3 and 4 switch their order between sparse and dense regimes. Mode 4 in the sparse regime seems to correspond to mode 3 in the dense and vice-versa. This suggests that the relative energy content in mode 3 compared to mode 4 is higher in one case and is lower in the other. The mode number where this change in mode shape occurs is named the "cut-off" mode and can be taken to represent the breakdown of the spatial similarity of the flow within the roughness sublayer. Note that this breakdown was not present in the full-field modes, where the 

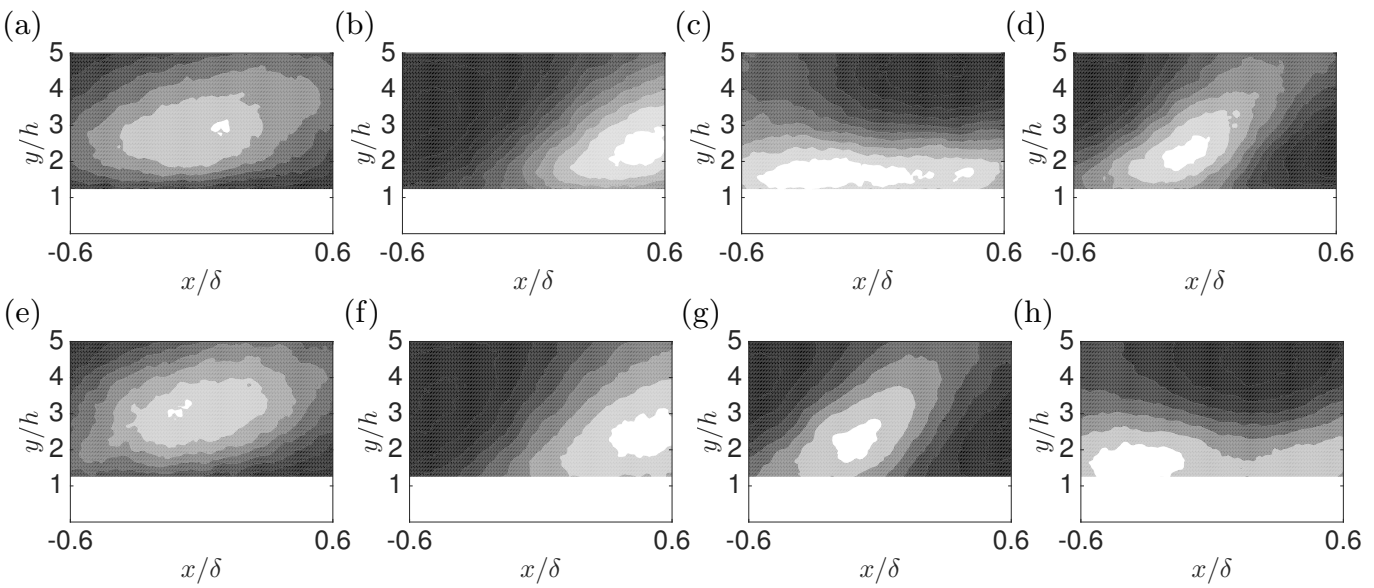

FiguRE 17. First four low order POD modes in the roughness sublayer for (a-d) sparse regime and $(\mathrm{e}-\mathrm{h})$ dense frontal solidity regime. Flow is left to right. POD modes are calculated on the combined $(u, v)$ field. Note that the aspect ratio of these figures have been changed to highlight the roughness sublayer. The wall-normal direction has been stretched compared to figures 15 and 16 .

energy in the outer region seemingly dominates the energy contained in the roughness sublayer. Physically, this means that a redistribution of energy across scales has taken place, therefore, the energy relevant scales are different in size/shape for the different regimes. An increase in frontal solidity results in redistributing the energy towards scales that are inclined to the wall (mode 3 compared to mode 4). A similar POD analysis for the plan solidity cases is shown in figure 18. It is noticeable that the mode shapes appear similar to previously discussed full-field cases and that for this solidity variation, the energy redistribution is absent. This suggests that there is a higher degree of spatial similarity in the energy containing motions in the roughness sublayer across the different plan solidities.

These findings are consistent with the hypothesis presented in $\S 3.1$. When the frontal solidity increases, the flow is progressively forced up and over the elements, hence a degradation of the local coherency is observed at high $\lambda_{F}$ values, introducing energy in to scales that are inclined to the wall, which is revealed by the appearance of the "cut-off" mode. This process is entirely absent for $\lambda_{P}$ variations as the frontal blockage is fixed, hence the cut-off mode does not appear in the RSL as the flow over the canopy is not significantly altered.

\subsubsection{Effect of surface morphology on energy distribution across scales}

Table 2 shows the fractional turbulent kinetic energy (FTKE) contribution $E_{i}$, of the $i$ th POD mode, $\phi_{i}$, to the total TKE for the $\lambda_{F}$ and $\lambda_{P}$ cases. It can be seen that cases with lower $\lambda_{F}$ tend to be characterised by lower energy content in the first POD mode. For example, mode 1 for the LF 1 case contains only $\approx 20 \%$ of the total energy, while for the LF3 and LF6 cases, its content reaches $\approx 22 \%$ and $\approx 25 \%$, respectively. This seems to suggest that the effect of an increased frontal solidity would be to redistribute the energy toward the lowest-order POD modes and therefore the larger-scales. It can also be inferred that the plan solidity cases present an opposite trend, where the first mode for the sparse case contains almost $26 \%$ of the total TKE while the contribution of the same mode for the medium and dense regimes (LP4 and LP6 cases for example) are only $21 \%$ and $22 \%$ respectively. 
22

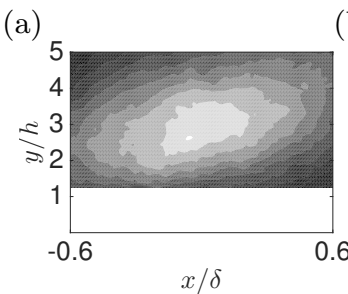

(e)

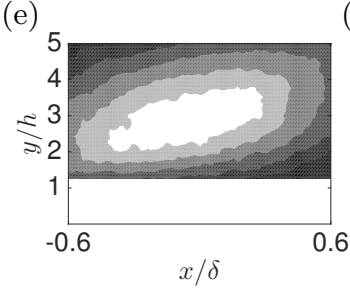

M. Placidi and B. Ganapathisubramani

(b)
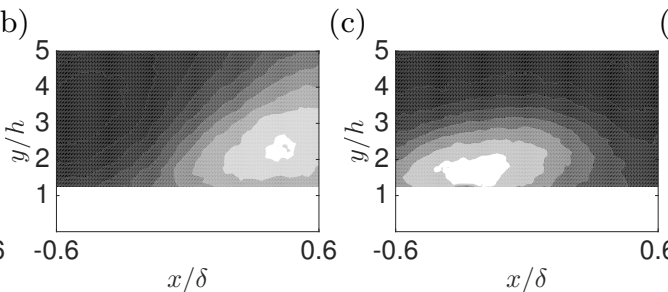

(f)

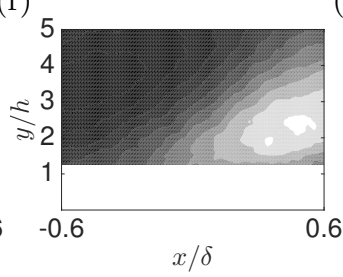

(g)

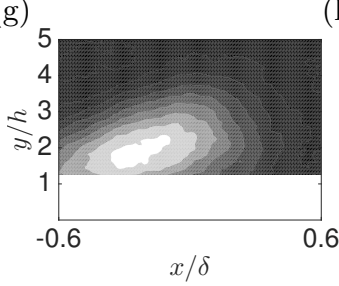

(d)

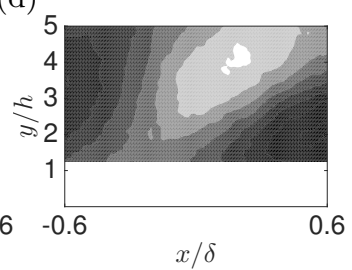

(h)

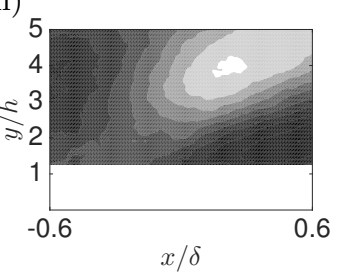

FiguRE 18. First four low order POD modes in the roughness sublayer for (a-d) sparse regime and $(\mathrm{e}-\mathrm{h})$ dense plan solidity regime. Flow is left to right. POD modes are calculated on the combined $(u, v)$ field.Note that the aspect ratio of these figures have been changed to highlight the roughness sublayer. The wall-normal direction has been stretched compared to figures 15 and 16 .

The cumulative turbulent kinetic energy (CTKE) is also presented in table 2. The CTKE of the first four modes contributes to $\approx 44 \%$ of the total TKE for the densest case, LF6, while it only represent contributions of $\approx 37 \%$ for the sparsest case, LF 1 . This further confirms that an increased frontal solidity results in a redistribution of energy towards lower modes. The opposite is true for the plan solidity cases. Similar trends can also be inferred from the number of modes necessary to contribute to the $50 \%$ of the total turbulent kinetic energy which is progressively lower for increased $\lambda_{F}$, while it increases for an increased $\lambda_{P}$. The reader's attention is also drawn on the fact that more than $\approx 600$ modes are needed to capture $95 \%$ of the resolved TKE from the $(x, y)$ plane PIV measurements in both cases. This reflects the complexity of these flows owing to the wide range of statistically important spatial scales present at these Reynolds numbers. These observations strongly suggest that the effect of an increased frontal solidity would be to redistribute the energy toward the highest energy POD modes. This could be an indication of an increase in coherency of the turbulent structures in the denser regimes. The opposite trend is found instead for the plan solidity variation. Increasing the $\lambda_{P}$ redistributes the energy toward the higher-order modes, hence the smaller scales.

\section{Conclusions}

Experiments were conducted in the fully-rough regime on surfaces consisting of distributed Lego ${ }^{\mathrm{TM}}$ bricks of uniform height, with $h / \delta \approx 0.1$. Measurements were made on six different surface morphologies with systematically increasing $\lambda_{F}$ at fixed $\lambda_{P}$ and six surfaces with varying $\lambda_{P}$ at fixed $\lambda_{F}$. This study examines the impact of surface morphology on wall-flows and could serve as benchmark data for validation of numerical simulations and other prediction models.

Results show, for the first time, the individual effect of frontal and plan solidities on various quantities in a controlled experiment. The non-dimensional roughness length reaches a peak value for $\lambda_{F}=0.21$, while it monotonically decreases with increasing $\lambda_{P}$. This is contrary to previous results obtained by examining the flow over cube roughness where the effects of $\lambda_{F}$ and $\lambda_{P}$ are coupled. This also suggests that morphometric studies 


\begin{tabular}{lcccccc} 
Dataset & $E_{1}$ & $E_{2}$ & $E_{3}$ & $E_{4}$ & $\sum_{i=1}^{4} E_{i}$ & $0.5 \sum_{i=1}^{n} E_{i}$ \\
\hline LF1 & 20 & 8 & 5 & 4 & 37 & 10 \\
LF2 & 22 & 9 & 6 & 4 & 41 & 8 \\
LF3 & 22 & 10 & 5 & 4 & 41 & 8 \\
LF4 & 23 & 9 & 5 & 4 & 41 & 8 \\
LF5 & 25 & 10 & 5 & 5 & 44 & 6 \\
LF6 & 25 & 9 & 5 & 5 & 44 & 6 \\
& & & & & & \\
LP1 & 26 & 10 & 5 & 4 & 45 & 6 \\
LP2 & 22 & 10 & 5 & 4 & 40 & 8 \\
LP3 & 19 & 8 & 8 & 4 & 39 & 9 \\
LP4 & 21 & 9 & 5 & 4 & 39 & 9 \\
LP5 & 22 & 8 & 5 & 4 & 39 & 9 \\
LP6 & 22 & 9 & 5 & 4 & 39 & 9 \\
& & & & & & \\
\hline
\end{tabular}

TABLE 2. Fractional TKE, $E_{i}$ and cumulative TKE $\sum_{i=1}^{n} E_{i}$ content versus mode number. $0.5 \sum_{i=1}^{n} E_{i}$ refers instead to the number of modes necessary to resolve the $50 \%$ of the turbulent kinetic energy contained in the flow. POD modes are calculated on the combined $(u, v)$ field only within the roughness sublayer.

purely based on the geometry of roughness elements might not prove accurate and further studies are required to identify appropriate correlations relating the geometry of the wall to its generated drag.

An investigation into the depth of the roughness sublayer has revealed different behaviours for variation in frontal and plan solidities. The RSL depth, however, is found to follow the same trend in both cases, i.e., it appears to be inversely proportional to the roughness length (or bulk drag). A decrease in drag is usually accompanied by a thickening of the the RSL and vice versa.

The use of proper orthogonal decomposition analysis to infer spatial similarity of flows over different wall morphologies was also demonstrated. The overall flow structure and the relative energy content across the different roughness morphologies appears to be very similar. However the relative energy content in some energy-containing modes within the roughness sublayer changes with increasing frontal solidity while it remains the same with increasing plan solidity.

Finally, the relative energy content in the POD modes strongly suggest that the effect of increasing $\lambda_{F}$ is to redistribute a larger proportion of the energy to the highest energy POD modes (i.e. the larger scales) while increasing $\lambda_{P}$ redistributes the energy toward the smaller scales (or higher-order POD modes).

\section{Acknowledgements}

We gratefully acknowledge the support from the European Research Council under the European Union's Seventh Framework Programme (FP7/2007-2013) / ERC Grant agreement No. 277472. We also thank Lloyd's Register Foundation (LRF) as well as the Faculty of Engineering and the Environment for supporting this research. 
Acharya, M, Bornstein, J \& Escudier, M P 1986 Turbulent boundary layers on rough surfaces. Experiments in Fluids 4, 33-47.

Adrian, R J, Christensen, K T \& Liu, Z C 2000 Analysis and interpretation of instantaneous turbulent velocity fields. Experiments in Fluids pp. 275-290.

Adrian, R J \& Westerweel, J 2011 Particle Image Velocimetry. Cambridge University Press.

Amir, M \& CAstro, I P 2011 Turbulence in rough-wall boundary layers: universality issues. Experiments in Fluids 51, 313-326.

Barros, Julio M \& Christensen, Kenneth T 2014 Observations of turbulent secondary flows in a rough-wall boundary layer. Journal of Fluid Mechanics $\mathbf{7 4 8}, \mathrm{R} 1$.

Benedict, L H \& Gould, R D 1996 Towards better uncertainty estimates for turbulence statistics. Experiments in Fluids 22, 129-136.

Berkooz, G, Holmes, P \& Lumley, J L 1993 The proper orthogonal decomposition in the analysis of turbulent flows. Annual Review of Fluid Mechanics 25, 539-575.

Castro, I P 2007 Rough-wall boundary layers: mean flow universality. Journal of Fluid Mechanics 585, 469-485.

Castro, I P, Segalini, A \& Alfredsson, P H 2013 Outer-layer turbulence intensities in smooth- and rough-wall boundary layers. Journal of Fluid Mechanics 727, 119-131.

Cheng, H \& CAstro, I P $2002 a$ Near-wall flow development after a step change in surface roughness. Boundary-Layer Meteorology 105, 411-432.

Cheng, H \& Castro, I P $2002 b$ Near wall flow over urban-like roughness. Boundary-Layer Meteorology 105, 411-432.

Cheng, H, Hayden, P, Robins, A G \& Castro, I P 2007 Flow over cube arrays of different packing densities. Journal of Wind Engineering and Industrial Aerodynamics 95 (8), 715740.

Coceal, O \& Belcher, S E 2004 A canopy model of mean winds through urban areas. Quarterly Journal of the Royal Meteorological Society 130 (599), 1349-1372.

Efros, V 2011 Scructure of turbulent boundary layer over a 2-D roughness. PhD thesis, Norwegian University of Science and Technology.

Flack, K A, Schultz, M P \& Connelly, J S 2007 Examination of a critical roughness height for outer layer similarity. Physics of Fluids 19 (9), 095104.

Flack, K A, Schultz, M P \& Shapiro, T A 2005 Experimental support for Townsend's Reynolds number similarity hypothesis on rough walls. Physics of Fluids 17 (3), 035102.

Grimmond, C S B \& Oke, T R 1999 Aerodynamic properties of urban areas derived, from analysis of surface form. Journal of Applied Meteorology 38, 1262-1292.

Hagishima, A, Tanimoto, J, Nagayama, K \& Meno, S 2009 Aerodynamic Parameters of Regular Arrays of Rectangular Blocks with Various Geometries. Boundary-Layer Meteorology 132, 315-337.

IyengAR, A K S \& FARELL, C 2001 Experimental issues in atmospheric boundary layer simulations: roughness length and integral length scale determination. Journal of Wind Engineering and Industrial Aerodynamics 89, 1059-1080.

JACKSON, P. S. 1976 The propagation of modified flow downstream of a change in roughness. Quart. J. Roy. Meteorol. Soc. 102, 924-933.

JACKSON, P. S. 1981 On the displacement height in the logarithmic velocity profile. Journal of Fluid Mechanics 111, 15-25.

Jimenez, J 2004 Turbulent flows over rough walls. Annual Review of Fluid Mechanics 36, 173196.

Kanda, M, Inagaki, A, Miyamoto, T, Gryschka, M \& RaAsch, S 2013 A New Aerodynamic Parametrization for Real Urban Surfaces. Boundary-Layer Meteorology 148 (2), 357-377.

KAnda, M, Moriwaki, R \& Kasamatsu, F 2004 Large-eddy simulation of turbulent organized structures within and above explicitly resolved cube arrays. Boundary-Layer Meteorology 112 (2), 343-368.

Krogstad, P A \& Efros, V 2010 Rough wall skin friction measurements using a high resolution surface balance. International Journal of Heat and Fluid Flow 31 (3), 429-433.

LEONARDi, S \& CASTRO, I P 2010 Channel flow over large cube roughness: a direct numerical simulation study. Journal of Fluid Mechanics 651, 519-539.

Leonardi, S, Orlandi, P, Smalley, R J, Djenidi, L \& Antonia, R A 2003 Direct numerical 
simulations of turbulent channel flow with transverse square bars on one wall. Journal of Fluid Mechanics 491, 229-238.

MACDONALD, R W 1998 An improved method for the estimation of surface roughness of obstacle arrays. Boundary-Layer Meteorology 97, 1857-1864.

Manes, C, Poggi, D \& Ridolfi, L 2011 Turbulent boundary layers over permeable walls: scaling and near-wall structure. Journal of Fluid Mechanics 687, 141-170.

Marusic, I., Monty, J P, Hultmark, M \& Smits, A J 2013 On the logarithmic region in wall turbulence. Journal of Fluid Mechanics 716, R3-1-R3-11.

Mejia-Alvarez, R \& Christensen, K T 2013 Wall-parallel stereo particle-image velocimetry measurements in the roughness sublayer of turbulent flow overlying highly irregular roughness. Physics of Fluids 25 (11), 115109.

Millward-Hopkins, J T, Tomlin, A S, Ma, L, Ingham, D \& Pourkashanian, M 2011 Estimating Aerodynamic Parameters of Urban-Like Surfaces with Heterogeneous Building Heights. Boundary-Layer Meteorology 141 (3), 467-490.

Nikuradse, J 1933 Laws of flow in rough pipes. NACA Tech. Memo. 1292 .

Pearson, D S, Goulart, P J \& Ganapathisubramani, B 2013 Turbulent separation upstream of a forward-facing step. Journal of Fluid Mechanics 724, 284-304.

Raffel, M., Willert, C. \& Kompenhans, J. 1998 Particle Image Velocimetry, 2nd edn. Berlin: Springer Verlag.

Raupach, M R, Antonia, R A \& Rajagopalan, S 1991 Rough-Wall Turbulent Boundary Layers. Applied Mechanics Reviews 44 (1), 1-25.

Reynolds, R T \& CAstro, I P 2008 Measurements in an urban-type boundary layer. Experiments in Fluids 45, 141-156.

Reynolds, R T, Hayden, P, Castro, I P \& Robins, A G 2007 Spanwise variations in nominally two-dimensionalrough-wall boundary layers. Experiments in Fluids 42, 311-320.

Santiago, J L, Coceal, O, Martilli, A \& Belcher, S E 2008 Variation of the Sectional Drag Coefficient of a Group of Buildings with Packing Density. Boundary-Layer Meteorology 128, 445-457.

Schlichting, H 1937 Experimental investigation of the problem of surface roughness. NACA TM 823, 1-60.

Schultz, M P \& Flack, K A 2005 Outer layer similarity in fully rough turbulent boundary layers. Experiments in Fluids 38 (3), 328-340.

Segalini, A, Örlü, R \& Alfredsson, P H 2013 Uncertainty analysis of the von Kármán constant. Experiments in Fluids 54, 1460.

Wu, Y \& Christensen, K T 2007 Outer-layer similarity in the presence of a practical roughwall topography. Physics of Fluids 19 (8), 085108. 